\title{
Onset of plume convection in mushy layers
}

\author{
By C. A. CHUNG AND FALIN CHEN \\ Institute of Applied Mechanics, National Taiwan University, Taipei, Taiwan 106, ROC
}

(Received 2 October 1998 and in revised form August 1999)

A weakly nonlinear analysis is employed to investigate the onset of plume convection in the mushy layer of a binary solution directionally solidified from below. An improved mathematical model including the constant pressure condition at the melt/mush interface is applied to analytically analyse the nonlinear behaviour of the convection. Results show that, due to the consideration of the constant pressure condition at the interface, the present analytical results are much better in comparison with the experiments than those obtained by previous studies, in which the no-vertical-flow condition at the interface was considered. It is also shown that the bifurcation to three-dimensional hexagon convection, corresponding to the onset of plume convection, is subcritical. For the case of small concentration ratio $\mathscr{C}$ (equation $(5 b)$ ) the channel of the plume is generated at the top of the mush and grows downwards into the mush. For the case of large $\mathscr{C}$, the channel may be generated at the interior of the mush and grow upwards to the top of the mush. The possible parameter regime in which the flow is of a stable down-centre hexagon is discussed.

\section{Introduction}

When a binary solution, such as ammonium chloride solution, is directionally solidified from below, a mushy layer forms between the melt region above and the solid region below due to the undercooling-induced morphological instability on the solidification front. During the solidification process, as a result of the rejection of lighter fluid upon solidification, several different kinds of convection have been observed in experiments. They include salt-finger convection, plume convection, bulk-fluid convection, and double-diffusive layer convection (see for example, Copley et al. 1970; Chen \& Chen 1991; Tait \& Jaupart 1992; Chen 1997). Most of the research efforts to date have studied salt-finger and plume convection, because of their potential relation to the formation of freckles, a highly undesirable feature deteriorating dramatically the mechanical properties of the casting of metallic alloy. The stability characteristics of salt-finger convection have been investigated theoretically by, for example, Worster (1992) and Chen, Lu \& Yang (1994) and experimentally by, for example, Tait \& Jaupart (1992). The stability features related to plume convection have been studied by, for example, Tait, Jahrling \& Jaupart (1992), Amberg \& Homsy (1993), Emms \& Fowler (1994), Worster \& Kerr (1994), Chen (1995), Chiareli \& Worster (1995), Anderson \& Worster (1995), and others. These investigations focused on the convection in the mush due to the fact that the plumes come directly out of the mush, leading to the inference that the driving mechanism of plume convection is internal to the mush. Recently, Worster (1997) has summarized the development of the relevant research in a review article, to which the readers may refer for more details.

Plume convection, which is an up-flow in a vertical narrow channel in the mush, is fundamentally a nonlinear phenomenon, as suggested by Fowler (1985), Worster 
(1992) and Chen et al. (1994) and recently confirmed by Amberg \& Homsy (1993) and Anderson \& Worster (1995). Tait et al. (1992) found from their experiment that, as plumes are observed, the convection is of a hexagonal planform, with down-flow at the centre (down-centre) and up-flow along the perimeters of the hexagon. The chimney is formed at the nodes where edges met, where the crystals are dissolved due to the interaction of convection and solidification. To study this nonlinear flow, Amberg \& Homsy (1993, hereafter denoted as A\&H) employed a weakly nonlinear approach to analyse the stability of both the two-dimensional roll and the three-dimensional hexagon convection in the mushy layer. They found that the two-dimensional roll bifurcates either supercritically or subcritically from the steady basic state, depending on the value of $K_{1}$ (cf. (22)), a parameter governing the change of the permeability with the solid fraction. The three-dimensional hexagon bifurcates transcritically with an up-flow at the centre of the hexagon (up-centre), which contradicts the experimental finding of Tait et al. (1992). Recently, Anderson \& Worster (1995, hereafter denoted as $\mathrm{A} \& \mathrm{~W}$ ) have tried to resolve this contradiction by considering the asymmetries of the system associated with the basic-state density curvature, higher-order variation of permeability, and interaction between temperature and solid fraction. They also chose different physical scales so that a more reasonable asymptotic expansion can be applied. They showed also that the three-dimensional hexagon bifurcates transcritically while both up-centre hexagons and down-centre hexagons can be stable depending on the control parameters of the system. Nevertheless, the parameter regime for the stable down-centre hexagon is found to be significantly different from the experimental value.

As pointed out by Worster (1997), a major shortcoming of the above two nonlinear analyses (i.e. $\mathrm{A} \& \mathrm{H}$ and $\mathrm{A} \& \mathrm{~W}$ ) is the use of the impermeable condition on the melt/mush interface, which allows no vertical flow to pass through the interface. Thus the mushy layer is completely isolated from the melt above, in spite of the fact that the dynamic coupling between the mush and the overlying melt can determine to a significant extent the stability characteristics of the flow in the mush. According to Worster, the use of the no-vertical-flow condition is expedient because, by doing so, the governing equations and associated boundary conditions can be solved analytically. In the present paper, we will show that, by considering the more realistic constant pressure condition, which allows a vertical flow through the melt/mush interface, the governing equations and associated boundary conditions are still analytically tractable.

The use of this constant pressure (or permeable) condition at the melt/mush interface leads to a great improvement to the analytical results. For example, we find that the parameter regime for the stable down-centre hexagon is possibly much closer to the experimental result than that of A\&W. Through an investigation into the variation of solid fraction, we find that the plume may be generated either at the top of the mush or in the interior of the mush, depending mostly on the concentration ratio $\mathscr{C}$, a parameter accounting for the ratio of the compositional difference between the solid and the solution to the compositional variation of the liquid of the mush. We also point out that the global stability behaviour of the present system is to some extent different from that of A\&W. This indicates that the permeable boundary condition on the melt/mush interface, as predicted by Worster (1997), plays a crucial role in determining the stability characteristics of the convection in the mush. We thus infer that, in order to capture completely the intrinsic characteristics of the convection in the mush of the directional solidification system, consideration of the influence of the flow of the overlying melt may be necessary. 


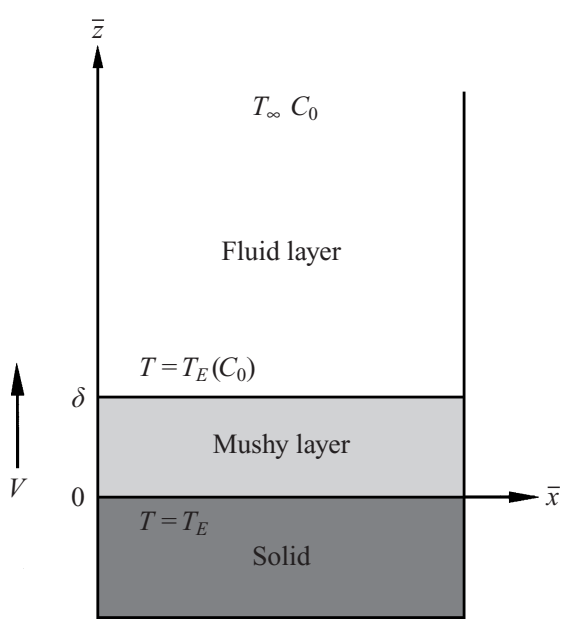

FIGURE 1. Schematic description of the physical system considered. The mushy layer is sandwiched between the fluid layer above and the solid layer below. The melt in the fluid layer is of concentration $C_{0}$ and temperature $T_{\infty}$ and the solid is of eutectic temperature $T_{E}$. The mush is of constant thickness, growing upwards into the melt with a constant speed $V$.

In the following, we describe in $\S 2$ the present physical system and the corresponding governing equations as well as the associated boundary conditions. In $\S 3$ we derive the basic state and the perturbation equations. In $\S 4$ we perform a weakly nonlinear analysis to investigate the stability of both the two-dimensional roll and the three-dimensional hexagon. In $\S 5$ we analyse the stability of the amplitude equations. In $\S 6$ we focus on the stability characteristics of the hexagon and discuss the factors influencing the sense of the flow at the centre of the hexagon. In $\$ 7$ the variation of solid fraction in the mush is considered. In $\S 8$ the global stability analysis is presented. In $\S 9$ a comparison of the critical Rayleigh numbers of the theoretical and experimental results is made. Finally, in $\S 10$ several concluding remarks are made.

\section{Problem description and formulation}

Consider the system shown in figure 1: a binary solution of concentration $C_{0}$ and temperature $T_{\infty}$ solidified from below. The mushy layer is sandwiched between the melt above and the eutectic solid below. Both the melt/mush and the mush/solid interfaces are assumed moving upwards with a constant velocity $V$. Assume that the solution concentration $C_{0}$ is greater than the eutectic concentration $C_{E}$ and the solution temperature $T_{\infty}$ is higher than the liquidus temperature $T_{L}\left(C_{0}\right)$. Assume also that the mushy layer is in a state of thermodynamic equilibrium so that the temperature and concentration of the mush satisfy the following liquidus relation:

$$
T=T_{L}\left(C_{0}\right)+\Gamma\left(C-C_{0}\right),
$$

where $\Gamma$ is the slope of the liquidus, assumed to be constant. The density of the fluid can then be written as

$$
\rho=\rho_{0}\left[1+\beta\left(C-C_{0}\right)\right],
$$

where $\beta=\beta^{*}-\Gamma \alpha^{*}, \alpha^{*}$ and $\beta^{*}$ are respectively thermal and solute expansion coefficients, and $\rho_{0}$ is the reference density.

We employ the following scales to render the equations dimensionless: velocity by 
$V$, length by $\kappa / V$, time by $\kappa / V^{2}$, and pressure by $\kappa \mu / \Pi(0)$. In the above scales, $\kappa$ is the thermal diffusivity, $\mu$ is the dynamic viscosity, and $\Pi(0)$ is the reference permeability. The permeability of the mush is a function of the solid fraction $\phi$, i.e. $\Pi=\Pi(\phi)$. The non-dimensional temperature (or concentration) can be written as

$$
\theta=\frac{T-T_{L}\left(C_{0}\right)}{\Delta T}=\frac{C-C_{0}}{\Delta C},
$$

where $\Delta T=T_{L}\left(C_{0}\right)-T_{E}$ and $\Delta C=C_{0}-C_{E}$, so that $\Delta T=\Gamma \Delta C$. Note from (3) that due to the liquidus relation (1) the temperature and concentration of the mush must be the same.

We employ a Galilean transformation that fixes the coordinates on the mush/solid interface. The non-dimensional governing equations in the mush are

$$
\begin{aligned}
\left(\frac{\partial}{\partial \bar{t}}-\frac{\partial}{\partial \bar{z}}\right)(\theta-\mathscr{F} \phi)+\boldsymbol{u} \cdot \bar{\nabla} \theta & =\bar{\nabla}^{2} \theta, \\
\left(\frac{\partial}{\partial \bar{t}}-\frac{\partial}{\partial \bar{z}}\right)[(1-\phi) \theta+\mathscr{C} \phi]+\boldsymbol{u} \cdot \bar{\nabla} \theta & =0, \\
K(\phi) \boldsymbol{u} & =-\bar{\nabla} p-R_{m} \theta \hat{\boldsymbol{e}}_{z}, \\
\bar{\nabla} \cdot \boldsymbol{u} & =0 .
\end{aligned}
$$

As discussed more fully below, governing equations in the melt are not required in the present analysis. Equations $(4 a)$ to $(4 d)$ are respectively the energy conservation equation, solute conservation equation, Darcy equation, and continuity equation. In them, the material properties of the solid and liquid phases are assumed to be identical, the solute diffusion is neglected because the solute diffusivity is very small, and the Boussinesq approximation is considered. The Stefan number $\mathscr{F}$, the concentration ratio $\mathscr{C}$ and the Rayleigh number $R_{m}$ are defined as

$$
\mathscr{F} \equiv \frac{\mathscr{L}}{c \Delta T}, \quad \mathscr{C} \equiv \frac{C_{s}-C_{0}}{\Delta C}, \quad R_{m} \equiv \frac{g \beta \Delta C \Pi(0) \kappa / V}{\kappa v},
$$

where $\mathscr{L}$ is the latent heat per unit volume, $c$ the specific heat per unit volume, $C_{s}$ the solid concentration, $g$ the gravitational acceleration, and $v$ the kinematic viscosity of the liquid. To understand the physical meaning as well as the influence of these three parameters, the reader may refer to, for example, Worster (1992) and Chen et al. (1994). Special notice should be given to the Rayleigh number $R_{m}$, which measures the ratio between the destabilizing compositional buoyancy and the stabilizing thermal diffusion, is a major parameter accounting for the stability of the system. The function $K(\phi)$ is defined as

$$
K(\phi)=\frac{\Pi(0)}{\Pi(\phi)},
$$

which describes the relation between the permeability and the solid fraction.

At the melt/mush interface, $\mathrm{A} \& \mathrm{H}$ and $\mathrm{A} \& \mathrm{~W}$ considered that the concentration remains at $C_{0}$ and the solid fraction is zero. To further simplify the problem, they assumed that no flow is allowed to penetrate through the interface. The mushy layer was accordingly isolated from the melt above. In the present study, we follow the derivation of Emms \& Fowler (1994) to decouple the mush from the melt. We assume that the Darcy number, a dimensionless parameter measuring the ratio of the average spacing between crystals of the mush to the characteristic length due 
to thermal diffusion, is small, and the pressure across the interface is continuous. Consequently, we obtain that the pressure is constant along the melt/mush interface, which is equivalent to the vertical gradient of the vertical velocity being zero. On the other hand, the mush/solid interface is considered as an impermeable, isothermal rigid boundary, as considered by $\mathrm{A} \& \mathrm{H}$ and $\mathrm{A} \& \mathrm{~W}$. These boundary conditions can then be written as

at $\bar{z}=0$

at $\bar{z}=\delta$

$$
\theta=-1, \quad w=0
$$

$$
\theta=0, \quad \frac{\partial w}{\partial \bar{z}}=0, \quad \phi=0,
$$

where $\delta$ is the dimensionless depth of the mushy layer. At $\bar{z}=0$ the boundary is of eutectic temperature and is impermeable. At $\bar{z}=\delta$, the temperature is the liquidus temperature, the vertical gradient of vertical velocity is zero (or pressure is constant), and the solid fraction is zero. The above equations are the same as those considered by $\mathrm{A} \& \mathrm{H}$ and $\mathrm{A} \& \mathrm{~W}$ except for $(8 b)$. We note that the position of the melt/mush interface is still fixed, being different from the reality that the interface is deformable.

\section{Basic state and perturbation equations}

We consider the near-eutectic approximation (Fowler 1985; A\&H; A\&W), assuming that $C_{0}$ is close to $C_{E}$ (so that $\mathscr{C}$ is large) and $\delta \ll 1$. We then let

$$
\mathscr{C}=\bar{C} / \delta,
$$

and assume $\bar{C}=O(1)$ as $\delta \rightarrow 0$. We also let

$$
\mathscr{F}=\bar{F} / \delta,
$$

and assume $\bar{F}=O(1)$ as $\delta \rightarrow 0$.

We follow A\&H to rescale the time and space based on the mushy-layer depth $\delta$ and define a new Rayleigh number $R$

$$
(\bar{x}, \bar{y}, \bar{z})=\delta(x, y, z), \quad \bar{t}=\delta^{2} t, \quad R^{2}=\delta R_{m} .
$$

The rescaled governing equations are

$$
\begin{aligned}
\left(\frac{\partial}{\partial t}-\delta \frac{\partial}{\partial z}\right)\left(\theta-\frac{\bar{F}}{\delta} \phi\right)+\delta \boldsymbol{u} \cdot \nabla \theta & =\nabla^{2} \theta, \\
\left(\frac{\partial}{\partial t}-\delta \frac{\partial}{\partial z}\right)\left[(1-\phi) \theta+\frac{\bar{C}}{\delta} \phi\right]+\delta \boldsymbol{u} \cdot \nabla \theta & =0, \\
\delta K(\phi) \boldsymbol{u} & =-\nabla p-R^{2} \theta \hat{\boldsymbol{e}}_{z}, \\
\nabla \cdot \boldsymbol{u} & =0 .
\end{aligned}
$$

Equations $(12 a-d)$ allow a motionless steady basic state solution, depending only on the vertical position. By assuming $\boldsymbol{u}=0$, we obtain from $(12 a-d)$ the following equations for the basic state:

$$
\frac{\mathrm{d}^{2} \theta_{B}}{\mathrm{~d} z^{2}}+\delta \frac{\mathrm{d} \theta_{B}}{\mathrm{~d} z}-\bar{F} \frac{\mathrm{d} \phi_{B}}{\mathrm{~d} z}=0,
$$




$$
\begin{aligned}
\bar{C} \frac{\mathrm{d} \phi_{B}}{\mathrm{~d} z}+\delta \frac{\mathrm{d} \theta_{B}}{\mathrm{~d} z}-\delta \frac{\mathrm{d}}{\mathrm{d} z}\left(\phi_{B} \theta_{B}\right) & =0, \\
\frac{\mathrm{d} P_{B}}{\mathrm{~d} z}+R \theta_{B} & =0,
\end{aligned}
$$

where the subscript $B$ denotes the basic state. The associated boundary conditions are

$$
\text { at } z=0
$$

$$
\theta_{B}=-1,
$$

at $z=1$

$$
\theta_{B}=0, \quad \phi_{B}=0 .
$$

The above equations can be solved by asymptotically expanding the dependent variables with respect to $\delta$, yielding

$$
\begin{aligned}
\theta_{B}=\theta_{B 0} & +\delta \theta_{B 1}+\cdots=(z-1)-\delta \frac{\Omega}{2}\left(z^{2}-z\right)+\cdots \\
\phi_{B}=\delta \bar{\phi}_{B} & =\delta \bar{\phi}_{B 0}+\delta^{2} \bar{\phi}_{B 1}+\cdots \\
& =-\delta \frac{z-1}{\bar{C}}+\delta^{2}\left[-\frac{(z-1)^{2}}{\bar{C}^{2}}+\frac{\Omega}{2 \bar{C}}\left(z^{2}-z\right)\right]+\cdots
\end{aligned}
$$

in which $\Omega=1+\bar{F} / \bar{C}$. Equations $(16 a, b)$ show that the assumption $\delta \ll 1$ renders a virtually linear temperature (or concentration) distribution and $\mathscr{C}=\bar{C} / \delta \gg 1$ renders a small solid fraction such that the permeability can be taken to be uniform. Because of the motionless state, the basic state solutions of (16) are the same as those of $\mathrm{A} \& \mathrm{~W}$, in spite of the use of the different boundary condition $(8 b)$ at the melt/mush interface.

The perturbation amplitude is measured by $\varepsilon$, so that the dependent variables can be written as

$$
\begin{aligned}
\theta & =\theta_{B}(z)+\varepsilon \hat{\theta}(x, y, z, t), \\
\phi & =\phi_{B}(z)+\varepsilon \hat{\phi}(x, y, x, t), \\
\boldsymbol{u} & =\varepsilon \frac{R}{\delta} \hat{\boldsymbol{u}}(x, y, z, t), \\
p & =R P_{B}(z)+R \varepsilon \hat{P}(x, y, z, t) .
\end{aligned}
$$

Meanwhile, we follow A\&W and define a 'slow time scale' $\tau$

$$
\tau=\varepsilon^{2} t
$$

such that the very slow development of the convection in the mush can be properly described. We note from (12) that, since $\boldsymbol{u}$ always appears together with $\delta$, it is therefore necessary to rescale $\boldsymbol{u}$ by $1 / \delta$, as shown in (17c). We substitute (17) into (12) and eliminate the basic state by using (13), yielding the perturbation equations as follows:

$$
\left(\delta \frac{\partial}{\partial z}-\varepsilon^{2} \frac{\partial}{\partial \tau}\right)\left(\hat{\theta}-\frac{\bar{F}}{\delta} \hat{\phi}\right)-R \theta_{B}^{\prime} \hat{w}+\nabla^{2} \hat{\theta}=\varepsilon R \hat{\boldsymbol{u}} \cdot \nabla \hat{\theta}
$$




$$
\begin{aligned}
\left(\delta \frac{\partial}{\partial z}-\varepsilon^{2} \frac{\partial}{\partial \tau}\right)\left[\left(1-\delta \bar{\phi}_{B}-\varepsilon \hat{\phi}\right) \hat{\theta}-\left(\theta_{B}-\frac{\bar{C}}{\delta}\right) \hat{\phi}\right]-R \theta_{B}^{\prime} \hat{w} & =\varepsilon R \hat{\boldsymbol{u}} \cdot \nabla \hat{\theta}, \\
\nabla^{2}(K \hat{w})-\frac{\partial}{\partial z}(\hat{\boldsymbol{u}} \cdot \nabla K)+R \nabla_{H}^{2} \hat{\theta} & =0, \\
\nabla^{2}(K \hat{u})-\frac{\partial}{\partial x}(\hat{\boldsymbol{u}} \cdot \nabla K)-R \frac{\partial^{2}}{\partial x \partial z} \hat{\theta} & =0, \\
\nabla^{2}(K \hat{v})-\frac{\partial}{\partial y}(\hat{\boldsymbol{u}} \cdot \nabla K)-R \frac{\partial^{2}}{\partial y \partial z} \hat{\theta} & =0, \\
\nabla \cdot \hat{\boldsymbol{u}} & =0,
\end{aligned}
$$

in which $\theta_{B}^{\prime}=\mathrm{d} \theta_{B} / \mathrm{d} z$ and $\nabla_{H}^{2}=\partial^{2} / \partial x^{2}+\partial^{2} / \partial y^{2}$. The perturbed boundary conditions at $z=0$ are

$$
\hat{\theta}=0, \quad \hat{w}=0,
$$

and at $z=1$ are

$$
\hat{\theta}=0, \quad \frac{\partial \hat{w}}{\partial z}=0, \quad \hat{\phi}=0 .
$$

Note that, except for (21b), equations (19), (20) and (21) are identical to those of $\mathrm{A} \& \mathrm{~W}$, and the basic states of the present model and A\&W are also the same.

Since both the basic-state solid fraction and the corresponding perturbation are small, we can expand the inverse of the permeability function $K(\phi)$ in a Taylor series with respect to the small solid fraction (A\&H). Namely, as $\phi \ll 1$, one has

$$
K(\phi)=1+K_{1} \phi+K_{2} \phi^{2}+\cdots .
$$

Since generally $K \propto(1-\phi)^{-m}$ where $m>0$ (Worster 1992; Emms \& Fowler 1994), implying that $K$ increases with $\phi$, only $K_{1}>0$ and $K_{2}>0$ are considered. As done by $\mathrm{A} \& \mathrm{~W}$, in order to study the higher-order nonlinear effects due to the temperature curvature, the non-uniform permeability, and the interaction between the temperature and the solid fraction on the flow stability, we assume $K_{1}=O(\delta)$. With this assumption, the slope of the transcritical bifurcation curve of critical Rayleigh number versus perturbation amplitude (as will be shown in figure 5) corresponding to a three-dimensional hexagon can change sign in accordance with the variation of the physical parameters. The details of the physical meaning of this assumption will be further discussed later.

\section{Weakly nonlinear analysis}

In (19) there are two small parameters, $\delta$ and $\varepsilon$. We follow the approach of A\&W, considering the formal asymptotic expansions in the double $\operatorname{limit}_{\lim _{\delta \rightarrow 0}}\left(\lim _{\varepsilon \rightarrow 0} f(\delta, \varepsilon)\right)$ for the function $f(\delta, \varepsilon)$; namely $\delta=O(1)$ as $\varepsilon \rightarrow 0$. The expansions of the perturbation quantities can be written as

$$
\begin{aligned}
\hat{\theta}=\left(\theta_{00}+\delta \theta_{01}+\cdots\right)+\varepsilon\left(\theta_{10}+\delta \theta_{11}+\cdots\right)+\varepsilon^{2}\left(\theta_{20}+\delta \theta_{21}+\cdots\right)+\cdots & \\
\Omega \hat{\phi}=\left(\phi_{00}+\delta \phi_{01}+\cdots\right)+\varepsilon\left(\phi_{10}+\delta \phi_{11}+\cdots\right) & \\
& +\varepsilon^{2}\left(\frac{1}{\delta} \phi_{2(-1)}+\phi_{20}+\delta \phi_{21}+\cdots\right)+\cdots
\end{aligned}
$$




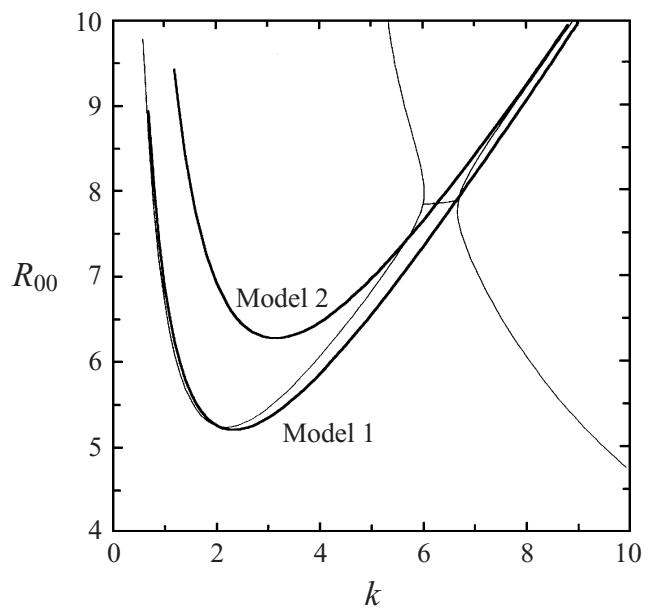

FIGURE 2. Neutral curves of $O\left(\varepsilon^{0} \delta^{0}\right)$ resulting from three different mathematical models. Thick curve-model 1: improved model of the present paper; thick curve-model 2: simplified model of Anderson \& Worster (1995); thin curve: two-layer model of Chen et al. (1994). Data are for 26\% ammonium chloride solution.

$$
\begin{aligned}
\Omega^{1 / 2} \hat{\boldsymbol{u}} & =\left(\boldsymbol{u}_{00}+\delta \boldsymbol{u}_{01}+\cdots\right)+\varepsilon\left(\boldsymbol{u}_{10}+\delta \boldsymbol{u}_{11}+\cdots\right)+\varepsilon^{2}\left(\boldsymbol{u}_{20}+\delta \boldsymbol{u}_{21}+\cdots\right)+\cdots, \\
\Omega^{1 / 2} R & =\left(R_{00}+\delta R_{01}+\cdots\right)+\varepsilon\left(R_{10}+\delta R_{11}+\cdots\right)+\varepsilon^{2}\left(R_{20}+\delta R_{21}+\cdots\right)+\cdots
\end{aligned}
$$

It is noteworthy that $(23 b)$ is singular at $O\left(\varepsilon^{2}\right)$ as $\delta \rightarrow 0$. This singular term comes from the forcing term (contributed by $\phi_{00}$ ) of the equation for $\hat{\phi}$ of $O\left(\varepsilon^{2}\right)$, indicating that $\hat{\phi}$ will be of $O\left(\varepsilon^{2} \delta^{-1}\right)$. It is therefore necessary to include the term $(1 / \delta) \phi_{2(-1)}$ at $O\left(\varepsilon^{2}\right)$ of $(23 b)$ to keep the equation in balance in terms of order of magnitude. More details will be discussed in $\S 4.5$. To render a meaningful asymptotic expansion of $(23 b)$, one needs $\varepsilon^{2} \delta \ll 1(\mathrm{~A} \& \mathrm{~W})$. In the following analysis we will focus on each order of the expansion of (23) to look into the stability characteristics of the two possible planforms: the two-dimensional roll and the three-dimensional hexagon.

\section{1. $O\left(\varepsilon^{0} \delta^{0}\right)$ solution}

By substituting (23) into (19)-(21), we obtain the perturbation equations at $O\left(\varepsilon^{0} \delta^{0}\right)$, see (A 1) in Appendix A. Note that (A 1) is a set of homogeneous equations and can be solved by separation of variables. The solutions are of the following form:

$$
\begin{aligned}
\theta_{00} & =\Theta_{00}(z) \eta_{0}(x, y, \tau), \quad \phi_{00}=\Phi_{00}(z) \eta_{0}(x, y, \tau), \\
w_{00} & =W_{00}(z) \eta_{0}(x, y, \tau), \quad u_{00}=U_{00}(z) \frac{\partial}{\partial x} \eta_{0}(x, y, \tau), \quad v_{00}=U_{00}(z) \frac{\partial}{\partial y} \eta_{0}(x, y, \tau) .
\end{aligned}
$$

$(24 c-e)$

The planar function (corresponding to the planform of convection) $\eta_{0}(x, y, \tau)$ satisfies

$$
\nabla_{H}^{2} \eta_{0}+k^{2} \eta_{0}=0 .
$$

The coefficient functions satisfy an eigenvalue problem, in which the eigenvalues are $R_{00}$ and $K$ the wavenumber. The value of $k$ will be fixed at its critical value once the neutral curve is obtained through (28), and thereafter $k$ is considered to be a fixed constant in the weakly nonlinear analysis. The solutions are obtained by substituting 
(a)

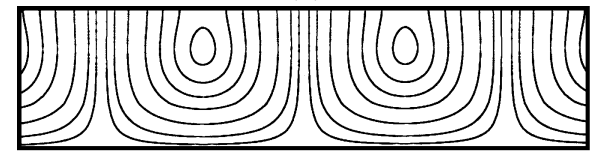

(b)

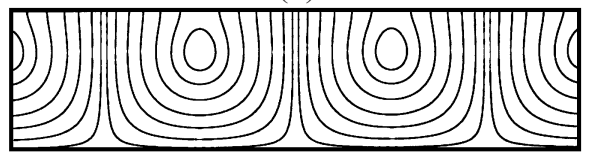

(c)

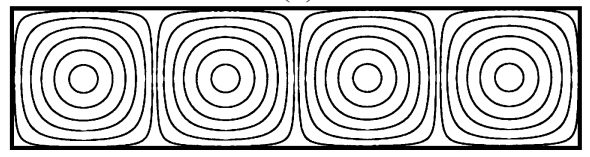

FiguRE 3. The flow patterns at onset corresponding to the critical points of the three neutral curves shown in figure 2. (a) Two-layer model, $\left(k, R_{00}\right)=(2.1802,5.2353)$ (Chen et al. 1994); (b) Model 1, $\left(k, R_{00}\right)=(2.3262,5.2055) ;(c)$ Model $2,\left(k, R_{00}\right)=(\pi, 2 \pi)$. It is noted that the difference between the flow patterns of model 1 and model 2 is only due to the boundary condition $(8 b)$. The wavelength of the convection cell is scaled on the mush depth.

(24) into (A 1) and use (25), yielding

$$
\begin{aligned}
\Theta_{00} & =-\left[\frac{\sin \left(q_{1} z\right)}{\sin \left(q_{1}\right)}-\frac{\sinh \left(q_{2} z\right)}{\sinh \left(q_{2}\right)}\right], \\
\Phi_{00} & =-\frac{k R_{00}}{\bar{C}}\left[\frac{\cos \left(q_{1} z\right)-\cos \left(q_{1}\right)}{q_{1} \sin \left(q_{1}\right)}-\frac{\cosh \left(q_{2} z\right)-\cosh \left(q_{2}\right)}{q_{2} \sinh \left(q_{2}\right)}\right], \\
W_{00} & =k\left[\frac{\sin \left(q_{1} z\right)}{\sin \left(q_{1}\right)}+\frac{\sinh \left(q_{2} z\right)}{\sinh \left(q_{2}\right)}\right], \quad U_{00}=\frac{1}{k}\left[\frac{q_{1} \cos \left(q_{1} z\right)}{\sin \left(q_{1}\right)}+\frac{q_{2} \cosh \left(q_{2} z\right)}{\sinh \left(q_{2}\right)}\right] .
\end{aligned}
$$

In above equations the parameters $q_{1}$ and $q_{2}$ are

$$
q_{1}=\sqrt{k R_{00}-k^{2}}, \quad q_{2}=\sqrt{k R_{00}+k^{2}},
$$

which satisfy the transcendental equation

$$
q_{1} \cot \left(q_{1}\right)+q_{2} \operatorname{coth}\left(q_{2}\right)=0 \text {. }
$$

There are an infinite number of solutions in terms of $k$ and $R_{00}$ satisfying (27) and (28), and each of them accounts for a neutral stability curve. The one of lowest $R_{00}$ is shown in figure 2, denoted 'model 1'. In this figure the curve denoted 'model 2' is the results we obtain by employing the model considered by A\&H and A\&W. The thin curve is the result obtained by Chen et al. (1994), who considered the two-layer model, i.e. a mushy layer underlying a fluid layer. All these three curves are computed for the $26 \%$ concentration ammonium chloride solution, whose physical properties are listed in Chen et al. It is seen that the use of the impermeable boundary condition at the melt/mush interface (model 2) renders a higher $R_{00}$, i.e. a more stable system, while the curves of model 1 and the two-layer model are fairly close. In fact, if the higher-order corrections are included, the curves of both model 1 and model 2 will move upwards a bit further, rendering an even smaller difference between model 1 and the two-layer model with a larger difference between model 1 and model 2 . Figure 3 illustrates the critical flow patterns corresponding to the three models. Again, 
the flow patterns of model 1 and the two-layer model are very similar, both having open streamlines which indicate that the flow of both the mush and the overlying melt are dynamically coupled through the interface. The flow pattern of model 2 is of smaller wavelength and the circulation is completely enclosed within the mush, reflecting the assumption that the mush is entirely isolated from the overlying melt. It is also noted that the flow pattern of model 2 is symmetric with respect to the centre height of the mush. This is due to the fact that to the present leading-order accuracy the permeability is uniform and the concentration gradient is linear in the mush.

The planar function $\eta_{0}$ of (25) can be written for a mode of wavenumber $k$ as

$$
\eta_{0}(x, y, \tau)=A_{1}(\tau) \cos (k y)+A_{2}(\tau) \cos \left[\frac{k(\sqrt{3} x+y)}{2}\right]+A_{3}(\tau) \cos \left[\frac{k(\sqrt{3} x-y)}{2}\right] .
$$

This equation accounts for the superposition of three perturbation modes. The phase of each mode differs from the other by $120^{\circ}$. When two of the three $A_{i}(\tau)$ are zero, for instance $A_{1} \neq 0$ and $A_{2}=A_{3}=0$, the flow is a two-dimensional roll. When $A_{1}=A_{2}=A_{3}$, the flow is a three-dimensional hexagon. The time-evolution equations of $A_{i}(\tau)$ which characterize the stability of the corresponding mode will be derived by the solvability conditions of the following higher-order analysis.

In the following analysis we will focus on the flow stability close to the critical point of the curve of model 1 , namely

$$
R_{00}=5.2055, \quad k=2.3262,
$$

to investigate the higher-order effects due to both the finite depth of the mush as well as the finite-amplitude perturbation on the stability of both the two-dimensional roll and three-dimensional hexagon. Note that the critical point obtained by A\&H and $\mathrm{A} \& \mathrm{~W}$ is located at $R_{00}=2 \pi$ and $k=\pi$, indicating that their system is more stable than either the present system or the two-layer system.

\section{2. $O\left(\varepsilon^{0} \delta\right)$ solution}

In this section we investigate the influence of the finite mush depth on the flow stability. The equations of $O\left(\varepsilon^{0} \delta\right)$ are given by (A 2) in Appendix A. These equations and those in the following sections are solved by the symbolic computation tool 'Mathematica'. By substituting (24), (26), (27), (29) and (30) into (A 2), one obtains a solution of the same form as (24). One needs only to change the index 00 into 01 and retain $\eta_{0}$ to get the solution. This solution actually contains only non-homogeneous terms since the homogeneous terms have been included in the $A_{i}(\tau)$ of $(29)$.

Equations (A $2 a$ ) and (A $2 c$ ) are employed to solve for $\theta_{01}$ and $w_{01}$ and the existence of the solution requires

$$
R_{01}=2.4930 \frac{\bar{F}}{\bar{C}^{2} \Omega}+0.1952 \Omega .
$$

The first term of the right-hand side of (31) is associated with the third term of the right-hand side of (A 2a), in which the interaction between the basic-state temperature and the perturbation of solid fraction is taken into account. The second term of the right-hand side of (31) mainly results from the consideration of the basic-state temperature curvature $\theta_{B 1}$ of (A $2 a$ ). The corrected Rayleigh number up to the present 
order, $O\left(\varepsilon^{0} \delta\right)$, is

$$
R=\Omega^{-1 / 2}\left(5.2055+\delta\left(2.4930 \frac{\bar{F}}{\bar{C}^{2} \Omega}+0.1925 \Omega\right)+\cdots\right) .
$$

Comparing with the result of $\mathrm{A} \& \mathrm{~W}$

$$
R=\Omega^{-1 / 2}\left(2 \pi+\frac{\delta \bar{F}}{\bar{C}^{2} \Omega}\left(\frac{4}{\pi}+\frac{\pi}{2}\right)+\cdots\right),
$$

one sees that (33) again results in a more stable system. Note that since $\Omega=1+\mathscr{F} / \mathscr{C}$, we have $\Omega=O(1)$.

\section{3. $O\left(\varepsilon \delta^{0}\right)$ solution}

In this subsection the effect of finite-amplitude perturbation on the flow stability is considered. We derive the perturbation equations of the present order, $O\left(\varepsilon \delta^{0}\right)$, and the next order, $O(\varepsilon \delta)$. The perturbation equations of $O\left(\varepsilon \delta^{0}\right)$ are given by (A 3$)$ in Appendix A. Due to the presence of the nonlinear advection term and the nonuniform permeability on the right-hand side of (A 3), there exists three perturbation modes corresponding to the three wavenumbers $k, \sqrt{3} k$ and $2 k$. The solutions of (A3) are of the following form:

$$
\begin{aligned}
& \theta_{10}=\Theta_{10}^{(c)} \eta_{1 c}+\Theta_{10}^{(0)} \eta_{10}+\Theta_{10}^{(1)} \eta_{11}+\Theta_{10}^{(2)} \eta_{12}, \\
& \phi_{10}=\Phi_{10}^{(c)} \eta_{1 c}+\Phi_{10}^{(0)} \eta_{10}+\Phi_{10}^{(1)} \eta_{11}+\Phi_{10}^{(2)} \eta_{12}, \\
& w_{10}=W_{10}^{(c)} \eta_{1 c}+W_{10}^{(0)} \eta_{10}+W_{10}^{(1)} \eta_{11}+W_{10}^{(2)} \eta_{12}, \\
& u_{10}=U_{10}^{(0)} \frac{\partial \eta_{10}}{\partial x}+U_{10}^{(1)} \frac{\partial \eta_{11}}{\partial x}+U_{10}^{(2)} \frac{\partial \eta_{12}}{\partial x}, \\
& v_{10}=U_{10}^{(0)} \frac{\partial \eta_{10}}{\partial y}+U_{10}^{(1)} \frac{\partial \eta_{11}}{\partial y}+U_{10}^{(2)} \frac{\partial \eta_{12}}{\partial y},
\end{aligned}
$$

where $\Theta_{10}, \Phi_{10}, W_{10}$ and $U_{10}$ are function of $z$, and

$$
\begin{gathered}
\eta_{1 c}(\tau)=\frac{A_{1}^{2}+A_{2}^{2}+A_{3}^{2}}{2}, \\
\eta_{10}(x, y, \tau)=A_{2} A_{3} \cos (k y)+A_{3} A_{1} \cos \left[\frac{k(\sqrt{3} x+y)}{2}\right]+A_{1} A_{2} \cos \left[\frac{k(\sqrt{3} x-y)}{2}\right], \\
\eta_{11}(x, y, \tau)=A_{2} A_{3} \cos (\sqrt{3} k x)+A_{3} A_{1} \cos \left[\frac{k(\sqrt{3} x-3 y)}{2}\right] \\
\eta_{12}(x, y, \tau)=A_{1}^{2} \cos \left[\frac{k(\sqrt{3} x+3 y)}{2}\right],
\end{gathered}
$$

in which the wavenumber of mode $\eta_{10}$ is $k$, of $\eta_{11}$ is $\sqrt{3} k$ and of $\eta_{12}$ is $2 k$. 
It is seen from (A 3 ) that $R_{10}$ appears as the coefficient of the forcing terms containing $w_{00}$ and $\theta_{00}$. The value of $R_{10}$ is therefore related to the form of the perturbation mode of $w_{00}$ and $\theta_{00}$. By comparing (29) and (35b), we can define three parameter $R_{10}^{(i)}, i=1,2,3$, corresponding to the three perturbation modes as follows:

$$
\begin{aligned}
& R_{10}=\frac{A_{2} A_{3}}{A_{1}} R_{10}^{(1)} \text { for } \cos (k y) \text { mode, } \\
& R_{10}=\frac{A_{3} A_{1}}{A_{2}} R_{10}^{(2)} \text { for } \cos \left[\frac{k(\sqrt{3} x+y)}{2}\right] \text { mode, } \\
& R_{10}=\frac{A_{1} A_{2}}{A_{3}} R_{10}^{(3)} \text { for } \cos \left[\frac{k(\sqrt{3} x-y)}{2}\right] \text { mode. }
\end{aligned}
$$

We substitute (34) and (36) into (A 3) to derive the solvability condition. From this condition we obtain the following two results:

(i) Two of the three $A_{i}$ are zero:

$$
R_{10}=0 \text { for } 2 \mathrm{D} \text { roll; }
$$

(ii) $\left|A_{1}\right|=\left|A_{2}\right|=\left|A_{3}\right|$ and $R_{10}^{(1)}=R_{10}^{(2)}=R_{10}^{(3)}=\bar{R}_{10}$ :

$$
\bar{R}_{10}=-22.7241 \frac{K_{1}}{\bar{C} \Omega} \text { for 3D hexagon. }
$$

The symbol || denotes the absolute value. The choice of the sign of the enclosed $A_{i}(\tau)$ is arbitrary since, by employing a shift of the coordinate in the horizontal direction, any combination of the sign of $A_{i}(\tau)$ can always be changed into the other. For example, we consider the combination $A_{1}=A, A_{2}=A_{3}=-A$ and substitute it into (29) to get a planar function. We then employ a shift of the coordinate $x_{1}=x-(2 / \sqrt{3} k) \pi$ and obtain a new planar function

$$
\eta_{0}=A \cos (k y)+A \cos \left[\frac{k\left(\sqrt{3} x_{1}+y\right)}{2}\right]+A \cos \left[\frac{k\left(\sqrt{3} x_{1}-y\right)}{2}\right],
$$

rendering $A_{1}=A_{2}=A_{3}=A$. We accordingly consider in the following only the case of $A_{1}=A_{2}=A_{3}$, which is representative of all the cases.

By comparing $(37 a, b)$ with those of $\mathrm{A} \& \mathrm{~W}$, we find that the present results are similar to theirs: $R_{10}=0$ for a two-dimensional roll and $R_{10}=-\left(3 \pi^{2} K_{1}\right) /(2 \bar{C})$ for a three-dimensional hexagon although we note that they omit a factor of $1 / \Omega$ in $\bar{R}_{10}$ (and also in $\bar{R}_{11}$ of (39b)). It is seen from (37) that, since $K_{1}, \bar{C}$ and $\Omega$ are positive, one has $\bar{R}_{10}<0$. This situation does not change if the physical parameters are varied, suggesting that it is therefore necessary to consider further the effect of higher-order, $O(\varepsilon \delta)$, on the bifurcation characteristics of hexagon convection.

\section{4. $O(\varepsilon \delta)$ solution}

In this subsection the effects due to both the finite depth of the mush and the finiteamplitude perturbation on the bifurcation characteristics of hexagon convection are investigated. The perturbation equations are (A 4) in Appendix A. The solutions of these equations are of the same form as (34), and can be obtained by simply changing the index 10 into 11 of all variables except $\eta$. Moreover, similarly to (36), we define 
the parameters $R_{11}^{(1)} \equiv\left(R_{11} A_{1}\right) /\left(A_{2} A_{3}\right), R_{11}^{(2)} \equiv\left(R_{11} A_{2}\right) /\left(A_{3} A_{1}\right), R_{11}^{(3)} \equiv\left(R_{11} A_{3}\right) /\left(A_{1} A_{2}\right)$, corresponding to the three different planar perturbation modes and substitute them into (A 4) to derive the solvability condition. The following two results can then be obtained by the solvability condition:

(i) Two of the three $A_{i}$ are zero:

$$
R_{11}=0 \text { for } 2 \mathrm{D} \text { roll; }
$$

(ii) $\left|A_{1}\right|=\left|A_{2}\right|=\left|A_{3}\right|$ and $R_{11}^{(1)}=R_{11}^{(2)}=R_{11}^{(3)}=\bar{R}_{11}$ :

$$
\bar{R}_{11}=-1.8643 \Omega+6.5116 \frac{\bar{F}}{\bar{C}^{2} \Omega}-27.1961 \frac{K_{2}}{\bar{C}^{2} \Omega}+K_{1} f\left(K_{1}\right) \text { for 3D hexagon. }
$$

In (39b), $f\left(K_{1}\right)$ is a first-order polynomial of $K_{1}$. Since $K_{1}$ is considered to be $O(\delta)$, there is no need to explicitly show the fourth term because it is smaller by an order of magnitude than the other terms. Equation (39b) illustrates the higher-order physical effects (A\&W), being accounted for by respectively the four terms on the right-hand side. First, the effect on the temperature curvature due to the upwardmoving freeing front is shown by the first term. Secondly, the influence imposed by the interaction between the temperature and the solid fraction is shown by the second term. Thirdly, the effect of the second-order solid fraction on the permeability (see (22)) is represented by the third term. Finally, the effect of the first-order solid fraction on the permeability is included in the fourth term.

Based on the correction at $O(\varepsilon)$ shown above, the corrected Rayleigh number can be written as $R_{\varepsilon}=\varepsilon\left(R_{10}+\delta R_{11}+O\left(\delta^{2}\right)\right)$, which is

(i) $R_{\varepsilon}=0$ for $2 \mathrm{D}$ roll;

(ii) $R_{\varepsilon}=\varepsilon A\left(\bar{R}_{10}+\delta \bar{R}_{11}+O\left(\delta^{2}\right)\right)$ for 3D hexagon.

In the following analysis at $O\left(\varepsilon^{2}\right)$ one will see that the correction of the Rayleigh number at $O\left(\varepsilon^{2}\right)$ is proportional to $(\varepsilon A)^{2}$. We accordingly find from $(23 d)$ that the slope of the bifurcation curve at $\varepsilon A=0$ can be written as $\mathrm{d} R /\left.\mathrm{d}(\varepsilon A)\right|_{\varepsilon A=0}=\Omega^{-1 / 2}\left(R_{\varepsilon} / \varepsilon A\right)$. Therefore (40) means, for the two-dimensional roll, the slope of the bifurcation curve is zero, i.e. $R_{\varepsilon} / \varepsilon A=0$. For the three-dimensional hexagon, due to the fact that $K_{1}>0$ and $\bar{R}_{10}<0$ (see (37b)) and if the contribution from the finite mushy-layer depth $\delta$ to $\bar{R}_{11}$ is ignored, the slope of the bifurcation curve must be negative, i.e. $R_{\varepsilon} / \varepsilon A<0$. To study the effect of $\bar{R}_{11}$ on the slope of the bifurcation, we follow A\&W by assuming the slope of the hexagon bifurcation to be small. This can be done by assuming $K_{1}=O(\delta)$ and

$$
K_{1}=\delta \frac{\bar{C} \Omega}{22.7241} \bar{R}_{11}+\delta \varepsilon \bar{K}_{1} .
$$

As a result, the correction to the Rayleigh number of the hexagon is

$$
R_{\varepsilon}=-(\varepsilon A)\left[\left(\frac{22.7241 \varepsilon \bar{K}_{1}}{\bar{C} \Omega}\right) \delta+O\left(\delta^{2}\right)\right] .
$$

This leads to a result that the slope $R_{\varepsilon} / \varepsilon A$ is small and proportional to $\varepsilon \bar{K}_{1}$, and the sign of $R_{\varepsilon} / \varepsilon A$ depends mainly on $\bar{K}_{1}$. Note that the value of $\bar{K}_{1}$ corresponds to the value of $K_{1},(41)$, and the other higher-order effects on $\bar{R}_{11}$ as well. As pointed out by A\&W, the purpose of (41) is twofold. First, the stable bifurcation of a threedimensional hexagon is close to that of a two-dimensional roll $\left(R_{\varepsilon}=0\right)$ so that same analysis procedure can be applied to the two different types of convection. Secondly, 


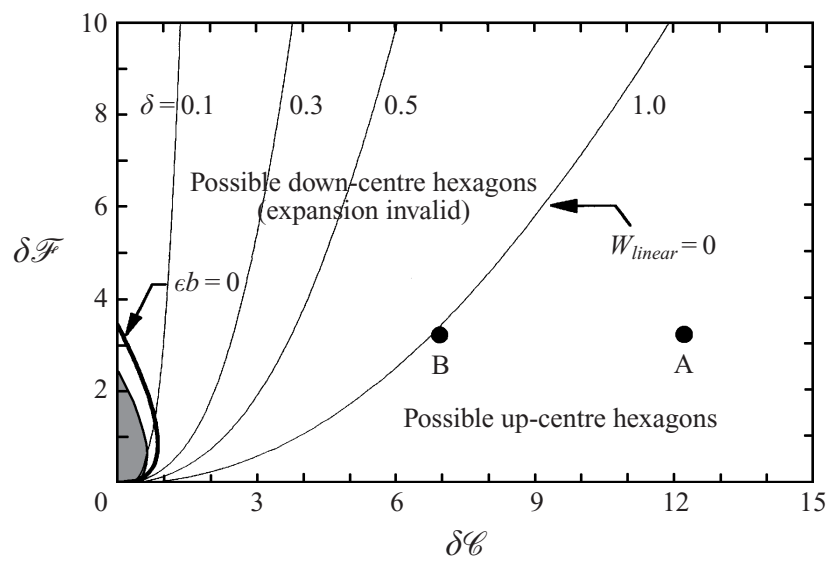

FIGURE 4. Regions of different three-dimensional hexagons when $K_{1}=0$ and $K_{2}=0$. The dark region in the lower-left corner is enclosed by the curve governed by equation (49), in which the oscillatory model is present. Several thin curves corresponding to different $\delta$ are shown. To the left of each thin curve, the sense of the flow at the hexagon centre is possibly downward, while to the right the flow at the hexagon centre is possibly upward. Note that $K_{1}=K_{2}=0$ is the extreme case where the curve $\varepsilon b=0$ is at the outermost position. Solid circle A: $26 \% \mathrm{NH}_{4} \mathrm{Cl}$; solid circle B: $30 \% \mathrm{NH}_{4} \mathrm{Cl}$.

the higher-order effect on $R_{11}$ is included, leading to the result that $R_{\varepsilon} / \varepsilon A$ may not be invariably negative so that the higher-order nonlinear effects must be considered.

We illustrate in figure 4 the regions of either positive or negative $R_{\varepsilon} / \varepsilon A$ of the three-dimensional hexagon. The second curve from the lower-left corner, denoted $\varepsilon b=0$, is for $R_{\varepsilon} / \varepsilon A=0$ (or $\bar{K}_{1}=0$ ). The region to the left of this curve is associated with $R_{\varepsilon} / \varepsilon A>0\left(\bar{K}_{1}<0\right)$ and that to the right with $R_{\varepsilon} / \varepsilon A<0\left(\bar{K}_{1}>0\right)$. In figure 4 both $K_{1}$ and $K_{2}$ are taken to be zero so that the region of $R_{\varepsilon} / \varepsilon A>0$ has the largest possible area. Note that the curve $R_{\varepsilon} / \varepsilon A=0\left(K_{1}=K_{2}=0\right)$ of A\&W is very close to the present one while their region of $R_{\varepsilon} / \varepsilon A>0$ is smaller. Figure 4 shows that the region of $R_{\varepsilon} / \varepsilon A<0$ is large, covering most of the cases of the experiments. This implies that the effect of the higher-order correction on the characteristics of hexagon convection is small since its influence is limited only to a small range of parameter in which $R_{\varepsilon} / \varepsilon A>0$. The thin curves of various $\delta$ and the label 'possible down-centre hexagons (expansion invalid)' will be explained in more detail later in $\S 6$.

\section{5. $O\left(\varepsilon^{2}\right)$ solution}

In the derivation of the equations at $O\left(\varepsilon^{2}\right)$, A\&W found that in the solute conservation equation there is a forcing term of $O(1 / \delta)$, taking into account the variation of solid fraction with time. The equations corresponding to $O\left(\varepsilon^{2} \delta^{-1}\right)$ are

$$
\frac{\partial \phi_{2(-1)}}{\partial z}=\frac{\partial \phi_{00}}{\partial \tau}
$$

and the corresponding boundary condition at $z=1$ is

$$
\phi_{2(-1)}=0 .
$$

The solution of (43) and (44) is

$$
\phi_{2(-1)}=\Phi_{2(-1)} \frac{\partial}{\partial \tau} \eta_{0}
$$


in which

$$
\begin{aligned}
\Phi_{2(-1)}= & -\frac{k R_{00}}{\bar{C}}\left[\frac{\sin \left(q_{1} z\right)}{q_{1}^{2} \sin \left(q_{1}\right)}-\frac{\sinh \left(q_{2}\right)}{q_{2}^{2} \sinh \left(q_{2}\right)}-\left(\frac{\cot \left(q_{1}\right)}{q_{1}}-\frac{\operatorname{coth}\left(q_{2}\right)}{q_{2}}\right) z\right. \\
& \left.-\frac{1}{q_{1}^{2}}+\frac{1}{q_{2}^{2}}+\frac{\cot \left(q_{1}\right)}{q_{1}}-\frac{\operatorname{coth}\left(q_{2}\right)}{q_{2}}\right] .
\end{aligned}
$$

The other equations at the present order are (A 5) and (A 6) in Appendix A. The solution at the present order contains five different modes, corresponding to the wavenumbers $k, \sqrt{3} k, 2 k, \sqrt{7} k$ and $3 k$. However, similarly to the previous lowerorder analysis, the values of $R_{20}$ and $R_{21}$ are associated only with the mode of $k$. Accordingly, for the convenience of solving these equations, we can let

$$
\begin{aligned}
\theta_{2}= & \Theta_{2}^{(1)}(z, \tau) \cos (k y)+\Theta_{2}^{(2)}(z, \tau) \cos \left[\frac{k(\sqrt{3} x+y)}{2}\right] \\
& +\Theta_{2}^{(3)}(z, \tau) \cos \left[\frac{k(\sqrt{3} x-y)}{2}\right]+\text { other modes, } \\
w_{2}= & W_{2}^{(1)}(z, \tau) \cos (k y)+W_{2}^{(2)}(z, \tau) \cos \left[\frac{k(\sqrt{3} x+y)}{2}\right] \\
& +W_{2}^{(3)}(z, \tau) \cos \left[\frac{k(\sqrt{3} x-y)}{2}\right]+\text { other modes. }
\end{aligned}
$$

In the above equations, $\psi_{2}=\psi_{20}+\delta \psi_{21}+\cdots$ and $\psi=\theta, w, \Theta^{(i)}, W^{(i)}$. We substitute (46) into the perturbation equations at $O\left(\varepsilon^{2}\right)$ and obtain the solvability condition by employing the corresponding boundary conditions. From the solvability condition the amplitude evolution equations of $A_{i}, i=1,2,3$, corresponding to the three modes $\cos (k y), \cos \lfloor k(\sqrt{3} x+y) / 2\rfloor$, and $\cos \lfloor k(\sqrt{3} x-y) / 2\rfloor$ are obtained as follows:

$$
\begin{aligned}
& a \dot{A}_{1}=b A_{2} A_{3}-c A_{1}^{3}-d A_{1}\left(A_{2}^{2}+A_{3}^{2}\right)+e A_{1} R_{2}, \\
& a \dot{A}_{2}=b A_{3} A_{1}-c A_{2}^{3}-d A_{2}\left(A_{3}^{2}+A_{1}^{2}\right)+e A_{2} R_{2}, \\
& a \dot{A}_{3}=b A_{1} A_{2}-c A_{3}^{3}-d A_{3}\left(A_{1}^{2}+A_{2}^{2}\right)+e A_{3} R_{2},
\end{aligned}
$$

in which $R_{2}=R_{20}+\delta R_{21}+\cdots$ and the coefficients of (47) are

$$
\begin{aligned}
& a=\Omega-2.4623 \frac{\bar{F}}{\bar{C}^{2} \Omega}+O(\delta), \quad b=\delta \bar{K}_{1}\left[22.7241 \frac{e}{\bar{C} \Omega}+O(\delta)\right], \\
& c=97.1604+1207.93 \frac{K_{2}}{\bar{C}^{2} \Omega^{2}}+O(\delta), \\
& d=167.024+2415.86 \frac{K_{2}}{\bar{C}^{2} \Omega^{2}}+O(\delta), \quad e=6.0146 .
\end{aligned}
$$

Except for the coefficients, (47) is of the same form as that of A\&W. Such equations can also be seen in a variety of other physical system, such as convection (Segel 1965), solidification (Brattkus \& Davis 1988), combustion (Kuske \& Matkowsky 1994), etc. 
The coefficients in (47) determine the stability characteristics of the system, as will be discussed in $\S 5$. In particular, it is worth noting the sign of $a$, since $a<0$ corresponds to the oscillatory instability (A\&W), which will be excluded from the present paper because only the stationary stability will be considered here. The region of $a<0$, as shown in figure 4 by the dark region in the lower-left corner, is described by the equation

$$
2.4623 \frac{\mathscr{F}}{\delta \mathscr{C}^{2}}<\left(1+\frac{\mathscr{F}}{\mathscr{C}}\right)^{2},
$$

which is obtained from (48a) for $a<0$. It is noted that this region covers only a small portion of the parameter range in terms of $\delta \mathscr{C}$ and $\delta \mathscr{F}$ while most of the cases considered in experiments are located in the region $a>0$.

\section{Analysis of the amplitude equations}

To analyse the amplitude equations (47), we employ a linear stability analysis to examine the stability characteristics of both the two-dimensional roll and the three-dimensional hexagon, as shown in the following.

\subsection{Two-dimensional roll stability}

We consider $\left(A_{1}, A_{2}, A_{3}\right)=(A, 0,0)$ as the equilibrium point and substitute it into (47), yielding the correction to the Rayleigh number

$$
R_{2}=\frac{c}{e} A^{2}
$$

To study the stability of the two-dimensional roll, we impose small perturbations with respect to the equilibrium point. According to the linear analysis, to ensure that the equilibrium point is stable, one requires

$$
|A|>\left|A^{(r)}\right|
$$

in which

$$
A^{(r)}=b /(d-c) .
$$

Equation (51) illustrates that the two-dimensional roll is stable when the perturbation amplitude in terms of $\varepsilon A$ is larger than $\varepsilon\left|A^{(r)}\right|$. Combining the results of $(23 d),(30 a)$, (31), (37a), (39a) and (50), the corrected critical Rayleigh number up to the present order is

in which

$$
R_{\text {rolls }}=R_{\text {linear }}+\Omega^{-1 / 2}\left[(\varepsilon A)^{2} c / e+\cdots\right]
$$

$$
R_{\text {linear }}=\Omega^{-1 / 2}\left(R_{00}+\delta R_{01}+O\left(\delta^{2}\right)\right) .
$$

We plot the variation of $R_{\text {rolls }}$ with respect to the perturbation amplitude $\varepsilon\left(A_{1}+\right.$ $\left.A_{2}+A_{3}\right)=\varepsilon A$ in figure 5 to illustrate the bifurcation curve of a two-dimensional roll. This curve is a concave parabolic facing to the right, having a zero slope, i.e. $\mathrm{d} R_{\text {rolls }} / \mathrm{d}(\varepsilon A)=0$, at $\varepsilon\left(A_{1}+A_{2}+A_{3}\right)=0$. When the $A$ of (53) is replaced by the $A^{(r)}$ of (52), the nonlinear critical Rayleigh number of a two-dimensional roll can be obtained as

$$
R^{(r)}=R_{\text {linear }}+\Omega^{-1 / 2}\left[\frac{(\varepsilon b)^{2} c}{e(d-c)^{2}}\right]
$$



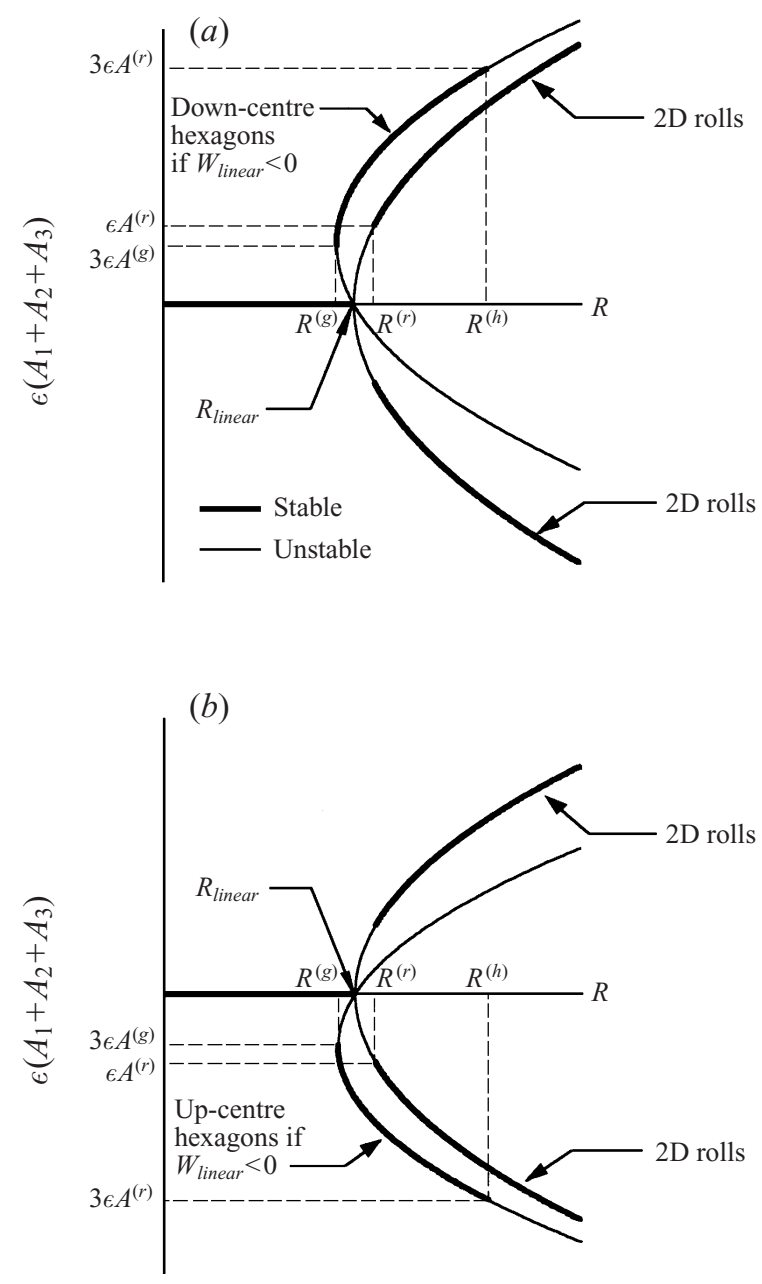

FIGURE 5. Bifurcation diagrams plotted as $\varepsilon\left(A_{1}+A_{2}+A_{3}\right)$ versus Rayleigh number. The thick curves are for the stable convection while the thin curves represent the unstable convection. $(a) \varepsilon b>0$ (i.e. $K_{1}>0, R / \varepsilon A<0$ ). The two-dimensional roll branch bifurcates supercritically and is stable when $R>R^{(r)}$. The three-dimensional hexagon branch bifurcates subcritically and becomes a stable down-centre cell when $R^{(h)}>R>R^{(g)}$ and $W_{\text {linear }}<0$. If $W_{\text {linear }}>0$, the cell becomes up-centre. (b) $\varepsilon b<0$ (i.e. $K_{1}<0, R / \varepsilon A>0$ ). The two-dimensional roll branch bifurcates supercritically and is stable when $R>R^{(R)}$. The three-dimensional hexagon branch bifurcates subcritically and becomes a stable up-centre cell when $R^{(h)}>R>R^{(g)}$ and $W_{\text {linear }}<0$. If $W_{\text {linear }}>0$, the cell becomes down-centre.

This shows that $R^{(r)}>R_{\text {linear }}$, so that the bifurcation of a two-dimensional roll is supercritical. Note that the curves of the two-dimensional rolls in figures $5(a)$ and $5(b)$ are the same.

\subsection{Three-dimensional hexagon stability}

We consider the equilibrium point $\left(A_{1}, A_{2}, A_{3}\right)=(A, A, A)$ and substitute this into (47), yielding

$$
R_{2}=\frac{(c+2 d)}{e} A^{2}
$$


Similar to the analysis of the two-dimensional roll, we impose a linear analysis on the equilibrium point to find its stability. To ensure a stable state, one requires

(i) $A^{(g)}<A<A^{(r)}$ as $b>0$,

(ii) $A^{(g)}>A>A^{(r)}$ as $b<0$,

in which $A^{(r)}$ is given by (52) and $A^{(g)}$ is

$$
A^{(g)}=\frac{b}{2(c+2 d)} .
$$

Consequently, the corrected Rayleigh number of a three-dimensional hexagon up to the present order is obtained by combining the results in $(23 d),(30 a),(31),(37 b)$, $(39 b)$ and (56), yielding

$$
R_{\text {hexagons }}=R_{\text {linear }}+\Omega^{-1 / 2}\left[-(\varepsilon A)\left(\frac{\varepsilon b}{e}\right)+(\varepsilon A)^{2}\left(\frac{c+2 d}{e}\right)+\cdots\right]
$$

in which $R_{\text {linear }}$ is given by (54). We plot in figure 5 the bifurcation curve of a threedimensional hexagon in terms of $R_{\text {hexagons }}$ versus $\varepsilon\left(A_{1}+A_{2}+A_{3}\right)=3 \varepsilon A$. In figure $5(a)$, where $\varepsilon b>0$ (i.e. $\bar{K}_{1}>0, R_{\varepsilon} / \varepsilon A<0$ ) is considered, the stable region lies in the quadrant $\varepsilon A>0$. Figure $5(b)$ depicts the case of $\varepsilon b<0$ (i.e. $\bar{K}_{1}<0, R_{\varepsilon} / \varepsilon A>0$ ), showing that the stable region lies in the quadrant $\varepsilon A<0$. The Rayleigh number corresponding to the amplitude $3 \varepsilon A^{(g)}$ is the global stability limit of the system, which can be obtained by replacing the $A$ of (59) by (58), yielding

$$
R^{(g)}=R_{\text {linear }}-\Omega^{-1 / 2}\left[\frac{(\varepsilon b)^{2}}{4 e(c+2 d)}\right] .
$$

It is seen that $R^{(g)}<R_{\text {linear }}$, indicating that the bifurcation of the three-dimensional hexagon is subcritical. The critical Rayleigh number corresponding to the amplitude $3 \varepsilon A^{(r)}$, beyond which the three-dimensional hexagon loses its stability, can be obtained by replacing the $A$ of (59) by (52), yielding

$$
R^{(h)}=R_{\text {linear }}+\Omega^{-1 / 2}\left[\frac{(\varepsilon b)^{2}(2 c+d)}{e(d-c)^{2}}\right] .
$$

\section{Sense of the flow at the hexagon centre}

Tait et al. (1992) found from their experiment using a $28 \% \mathrm{NH}_{4} \mathrm{Cl}$ solution that the three-dimensional hexagon is a convection of down-flow at the centre and of up-flow along the perimeters of the hexagon. To investigate this feature, $A \& H$ considered the $K_{1}$ effect and found that the hexagon is of up-flow at the centre, quite the opposite to the experimental result. A\&W extended the analysis of $A \& H$ by including the asymmetry of the system associated with the density curvature, higher-order variation of permeability, interaction between temperature and solid fraction, etc. They identified a small parametric regime in which the down-centre hexagon is stable. Nevertheless, this regime is still very different from the conditions in terms of $\mathscr{C}$ and $\mathscr{F}$ of the experiment of Tait et al. (1992). They attributed this discrepancy to the use of the impermeable boundary condition at the melt/mush interface. This conjecture is examined in the present paper, in which the constant pressure condition is used to replace the impermeable condition. The details are discussed in the following. 
To examine the sense of the flow at the centre of a hexagon cell, it is necessary to investigate the vertical velocity $w$, which can be derived from $(17 c),(23 c)$ and $(24 c)$ as

where

$$
w=\left(\frac{R}{\Omega^{1 / 2} \delta}\right)\left[\varepsilon W_{\text {linear }}(z) \eta_{0}(x, y, \tau)+O\left(\varepsilon^{2}\right)\right],
$$

$$
W_{\text {linear }}(z)=W_{00}(z)+\delta W_{01}(z)+O\left(\delta^{2}\right),
$$

and $W_{00}(z)$ is given by $(26 c)$. By solving (A $\left.2 a\right)$ and (A $\left.2 c\right)$ with the symbolic computational tool Mathematica, we obtain $W_{01}(z)$ as

$$
\begin{aligned}
W_{01}(z)= & \alpha^{(0)}+\left(\alpha_{c}^{(0)}+\alpha_{c}^{(1)} z+\alpha_{c}^{(2)} z^{2}\right) \cos \left(q_{1} z\right)+\left(\alpha_{s}^{(0)}+\alpha_{s}^{(1)} z\right) \sin \left(q_{1} z\right) \\
& +\left(\alpha_{c h}^{(0)}+\alpha_{c h}^{(1)} z+\alpha_{c h}^{(2)} z^{2}\right) \cosh \left(q_{2} z\right)+\left(\alpha_{s h}^{(0)}+\alpha_{s h}^{(1)} z\right) \sinh \left(q_{2} z\right) .
\end{aligned}
$$

The coefficients are shown in Appendix B. The eigenvalues $q_{1}$ and $q_{2}$ of (63) can be obtained through (27) by considering $R_{00}=5.2055$ and $k=2.3262$ as $q_{1}=2.5880$ and $q_{2}=4.1857$. From Appendix B, it is seen that the coefficients can be divided into two groups. One consists of the terms containing $\bar{F} / \bar{C}^{2} \Omega$, which are the result of the interaction between the basic-state temperature and the solid fraction, as shown by the third term on the right-hand side of (A $2 a$ ). The other consists of the terms containing $\Omega$, which stem from the effects of both the basic-state temperature curvature $\theta_{B 1}$ and the vertical gradient of the first-order temperature perturbation $\theta_{00}$, as shown by respectively the first term containing $\theta_{\mathrm{B} 1}^{\prime}$ and the second term on the right-hand side of (A $2 a$ ).

For a three-dimensional hexagon, we have $A_{i}=A, i=1,2,3$. The planar function (29) can be written as

$$
\eta_{0}(x, y, \tau)=A(\tau)\left\{\cos (k y)+\cos \left[\frac{k(\sqrt{3} x+y)}{2}\right]+\cos \left[\frac{k(\sqrt{3} x-y)}{2}\right]\right\} .
$$

Assume that the centre of the hexagon is located at $(x, y)=(0,0)$, then $\eta_{0}(0,0, \tau)=$ $3 A(\tau)$. The vertical velocity of the centre is thus

$$
w_{\text {centre }}=\frac{3 \varepsilon R}{\Omega^{1 / 2} \delta}\left[A(\tau) W_{\text {linear }}(z)\right]+O\left(\varepsilon^{2}\right) .
$$

It is seen from (65) that the sign of $w_{\text {centre }}$ depends on the sign of the product of $A(\tau)$ and $W_{\text {linear }}$. The sign of $A(\tau)$ can be determined from figure 5: as $\varepsilon b>0, A(\tau)>0$ for a stable hexagon; as $\varepsilon b<0, A(\tau)<0$ for a stable hexagon. Once the sign of $W_{\text {linear }}$ is known, the sense of the flow at the centre of the hexagon can be determined as follows. For $\varepsilon b>0$ (i.e. $\bar{K}_{1}>0, R_{\varepsilon} / \varepsilon A<0$ ) as shown in figure $5(a)$, the hexagon is stable only when $\varepsilon A>0$. Accordingly, as can be seen from (65), the hexagon is a down-centre flow as $W_{\text {linear }}<0$ and is an up-centre flow as $W_{\text {linear }}>0$. For $\varepsilon b<0$ (i.e. $\bar{K}_{1}<0, R_{\varepsilon} / \varepsilon A>0$ ), as shown in figure $5(b)$, the hexagon can be stable only when $\varepsilon A<0$, so that it is an up-centre flow as $W_{\text {linear }}<0$ and is a down-centre flow when $W_{\text {linear }}>0$.

To examine the sign of $W_{\text {linear }}$ at the top of the mush, we substitute $z=1$ into (62b), yielding

$$
W_{\text {linear }}(1)=4.6524-\delta\left(1.4104 \Omega+54.0076 \frac{\bar{F}}{\bar{C}^{2} \Omega}\right) .
$$

It is noted that the nature of $(62 b)$ requires that the series expansion is valid 
asymptotically only as $\delta$ approaches zero. To apply the present analysis to a real case, a finite value of $\delta$ must be considered. Consequently, the validity of (66) becomes questionable. It is seen from (66) that $W_{00}(1)$ contributes only the positive value 4.6524 to $W_{\text {linear }}$. As a result, to render a negative $W_{\text {linear }}, W_{01}$ must be negative and of the same magnitude as $W_{00}$ when $\delta$ is of a finite value. This implies that the asymptotic expansion of $(66)$ up to $O(\delta)$ is not accurate enough if $W_{\text {linear }}<0$. Namely, the asymptotic expansion of (62b) may not be a convergent series if $\delta$ is finite and $W_{\text {linear }}<0$. As we tried to compute $W_{02}$ for a special case (which is extremely tedious), we found that there is a trend that the sign of $\left(W_{00}(z), W_{01}(z), W_{02}(z), W_{03}(z), \ldots\right)$ is $(+,-,+,-, \ldots)$. This indicates that to obtain a negative $W_{\text {linear }}$, the expansion of (62b) up to $O(\delta)$ may not be accurate enough to reflect the fact; namely higher-order terms are required to reach a conclusive result. To obtain a positive $W_{\text {linear }}$ of a case of finite $\delta$, on the other hand, $(62 b)$ may be good enough to indicate qualitatively the sense of the flow at the hexagon centre because $\left|W_{00}\right|>\left|W_{01}\right|$, and this tendency seems to apply to higher-order terms.

To the best of our knowledge, it is virtually impossible to solve $W_{02}, W_{03}, \ldots$ by present mathematical tools and (66) may be the best result we can have currently. We therefore employ (66) to discuss the sense of the flow at the hexagon centre, and the results are summarized in figure 4. Based on (66), we illustrate in figure 4 several curves of $W_{\text {linear }}=0$ corresponding to various $\delta$. The region on the right of the curve accounts possibly (due to inaccurate expansion) for $W_{\text {linear }}(1)>0$ when the accuracy up to $O(\delta)$ is considered, implying that in this parameter regime the convection is possibly of an up-centre hexagon. In the region on the left of the curve it is possible that $W_{\text {linear }}(1)<0$ and the convection is possibly of a down-centre hexagon. Due to the insufficient expansion of the series of $(62 b)$, we mark the two regions divided by the $\delta$ curve as respectively 'possible down-centre hexagon/expansion invalid' and 'possible up-centre hexagon'.

In figure 4 the region enclosed by the two curves denoted $\delta$ and $\varepsilon b=0$ is that in which a stable down-centre hexagon may possibly exist. The region decreases its area with decreasing $\delta$. As $\delta=0.1$, part of the region $\varepsilon b<0$ (i.e. $R_{\varepsilon} / \varepsilon A>0$ ) becomes that of a possible down-centre hexagon. As $\delta$ approaches zero, the region of a possible down-centre hexagon is virtually completely covered by $\varepsilon b<0$. In other words, the down-centre hexagon can be stable in the region $R_{\varepsilon} / \varepsilon A>0$, consistent with the prediction of $\mathrm{A} \& \mathrm{~W}$. To give a hint regarding the conditions considered in experiments, we also show in figure 4 two solid circles denoted by A and B, corresponding respectively to the cases of $26 \%(\mathscr{F}=3.2, \mathscr{C}=12.3, \delta=1)$ and $30 \%$ ammonium chloride solution $(\mathscr{F}=3.2, \mathscr{C}=7, \delta=1)$.

The possible divergence of the series and the tendency of the negative correction term of $O(\delta)$ may give a clue that the features of the flow in the solidifying mush can differ from those of a non-reacting porous layer. Through the interaction among the solidification, the porosity and the resultant active non-uniform permeability, all of these appear in the correction terms of finite mush depth; an active mushy layer may consequently allow a down-flow at the centre of the hexagon. This may merit further research in which the near-eutectic approximation, which assumes $\delta \ll 1$, is lifted.

\section{Variation of solid fraction in the mush}

The solid fraction is another interesting factor to be considered for its influence on the characteristics of the three-dimensional hexagon. By doing some rearrangements of $(17 b),(23 b)$ and $(24 b)$ and by neglecting higher-order terms, the solid fraction $\phi$ 


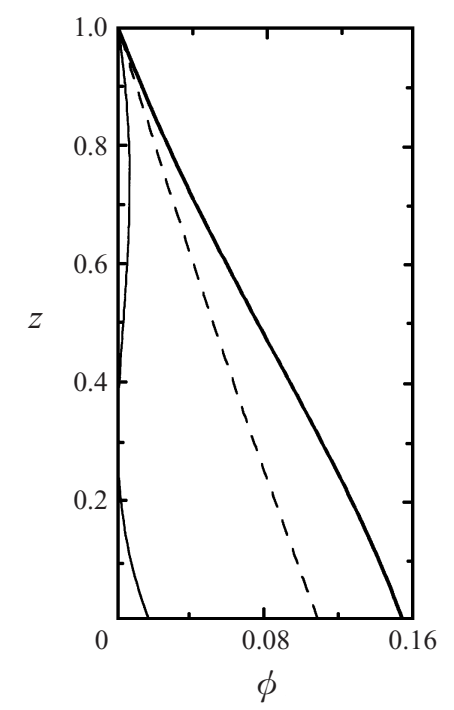

FIGURE 6. The vertical distribution of $\phi$ along the centreline (thin curve), along the node-line (thick curve), and of the basic state (dashed curve), which is horizontally independent. For this case, $\mathscr{F}=3, \delta=0.3, \mathscr{C}=8.0$ and $\varepsilon A=0.014$. Note that the value of $\varepsilon A$ is the maximum amplitude beyond which the $\phi$ will become negative.

can be derived as

$$
\phi=\phi_{B}+\left(\frac{\varepsilon A}{\Omega}\right)\left(\Phi_{00}+\delta \Phi_{01}\right) \eta_{0}+\cdots
$$

in which $\phi_{B}$ is given by (16b), $\Phi_{00}$ by (26b), and $\eta_{0}$ by (64). The function $\Phi_{01}$ can be obtained by solving (A $2 a-c)$ with a similar procedure to $\S 4.1$, yielding

$$
\begin{aligned}
\Phi_{01}(z)= & \beta^{(0)}+\beta^{(1)} z+\left(\beta_{c}^{(0)}+\beta_{c}^{(1)} z\right) \cos \left(q_{1} z\right)+\left(\beta_{s}^{(0)}+\beta_{s}^{(1)} z+\beta_{s}^{(2)} z^{2}\right) \sin \left(q_{1} z\right) \\
& +\left(\beta_{c h}^{(0)}+\beta_{c h}^{(1)} z\right) \cosh \left(q_{2} z\right)+\left(\beta_{s h}^{(0)}+\beta_{s h}^{(1)} z+\beta_{s h}^{(2)} z^{2}\right) \sinh \left(q_{2} z\right) .
\end{aligned}
$$

The coefficients, as shown in Appendix C, are similar to those shown in Appendix $\mathrm{B}$, stemming from the same physical mechanisms driving the form of the coefficients of $W_{01}$ (equation (63)). Note that the series expansion in (67) may have a similar problem of slow-convergent rate as that in $(62 b)$. In the following we will focus on the cases corresponding to the possible up-centre hexagon because the expansion can be more accurate.

Based on (67), we examine the vertical distribution of $\phi$ at both the centre point and the node point of the hexagon. The results are shown in figure 6. In this figure, the dashed curve accounts for the basic state $\phi_{B}$, the thin curve for the $\phi$ along the vertical line through the centre point (the centreline) and the thick curve for the $\phi$ along the vertical line through the node point (the node-line). In figure $6, \delta=0.3$, $\mathscr{F}=3$ and $\mathscr{C}=8.0$, and the perturbation amplitude is $\varepsilon A=0.014$. This value of $\varepsilon A$ is selected so that $\phi$ reaches its positive minimum. In other words, as the perturbation is larger than these $\varepsilon A$, the solid fraction will become negative. Physically, it means that the solid dissolves and the plume channel forms (A\&H).

The case of figure 6 lies in the region corresponding to a possible up-centre hexagon. The solid fraction along the centreline decreases under the perturbation and its minimum occurs in the interior of the mush, implying that the channel may be 
initiated from the interior of the mush. We note that the result of figure 6 is similar to that obtained by Schulze \& Worster (1999, figure 5) in which the nonlinear twodimensional roll convection was computed numerically and showed that the plume may possibly be initiated within the mush. We noted also from the calculations of other relevant cases that the minimum solid fraction occurs at the top of the mush when $\mathscr{C}$ is small, but occurs in the interior of the mush when $\mathscr{C}$ is larger. It can be seen from the basic-state solid fraction of (16b) that, although the solid fraction at the top is always the minimum, the vertical gradient of the solid fraction increases when $\mathscr{C}$ is smaller, so that for a larger $\mathscr{C}$ the vertical gradient of solid fraction is small, resulting in a mush of virtually uniformly small solid fraction. After being perturbed, accordingly, the opportunity for the generation of the chimney from the interior of the mush is higher.

\section{Global stability analysis}

To investigate the global stability of the system, the values of both $R_{\text {linear }}$ and $R^{(g)}$ for various $\delta, \mathscr{F}, K_{1}$ and $\mathscr{C}$, are computed by employing respectively (54) and (60), and the results are shown in figure 7. For each case shown in figure 7, three of the four parameters are fixed and one varies, while $K_{2}=0$. Results show that, except figure $7(c)$ regarding the influence of $\delta$, the effects due to varying $\mathscr{F}, K_{1}$ and $\mathscr{C}$ on the stability of the system are similar to those obtained by A\&W, to which the reader may refer for the physical background. By and large, for all the cases considered $R_{\text {linear }}$ is invariably larger than $R^{(g)}$, indicating that the system is always of subcritical instability in spite of the varying physical parameters.

The influence of $\delta$ found by the present study is however different from that of $\mathrm{A} \& \mathrm{~W}$, which is explained in the following. Figure $7(c)$ shows that $R_{\text {linear }}$ increases with $\delta$ constantly, contradicting the result of A\&W which showed that $R_{\text {linear }}$ remains constant in spite of varying $\delta$. This discrepancy stems from the Rayleigh number correction due to finite $\delta$ (equation (31)) which can be rewritten as $\delta R_{01}=2.4930 \mathscr{F} / \mathscr{C}^{2} \Omega+0.1925 \Omega \delta$. It is the term $0.1925 \Omega \delta$ which makes $R_{\text {linear }}$ increase linearly with $\delta$. This term results from the effects due to the asymmetry of the boundary conditions and the temperature curvature. With regard to the variation of $R^{(g)}$, it essentially follows the trend of $R_{\text {linear }}$ except near the two ends of the curve, i.e. $\delta=0$ and $\delta=1$. Near $\delta=0$ the deviation of $R^{(g)}$ from $R_{\text {linear }}$ is due to the fact that $\bar{C}=\delta \mathscr{C} \rightarrow 0$ as $\delta \rightarrow 0,(37 b)$, augmenting the effect due to the variation of permeability. Near $\delta=1$, according to the first term on the right-hand side of $(39 b)$, the effect due to the temperature curvature leads to the deviation of the two Rayleigh numbers. It is noteworthy that except for $\delta \rightarrow 0$ the slope increment of $R^{(g)}$ is very close to that of $R_{\text {linear }}$, which is approximately $0.1925 \Omega$, and so is less than unity. Consequently, the mushy-layer Rayleigh number $R_{m}^{(g)}$ decreases with increasing $\delta$ unless $\delta \rightarrow 0$ (equation (11c)), suggesting that the system is less stable as $\delta$ increases.

\section{Comparison of critical Rayleigh numbers}

In the experiment using the $28 \%$ ammonium chloride solution, Tait et al. (1992) (TJJ) studied the stability of plume convection by decreasing gradually the cooling temperature at the mush/solid interface and determined the critical Rayleigh number 

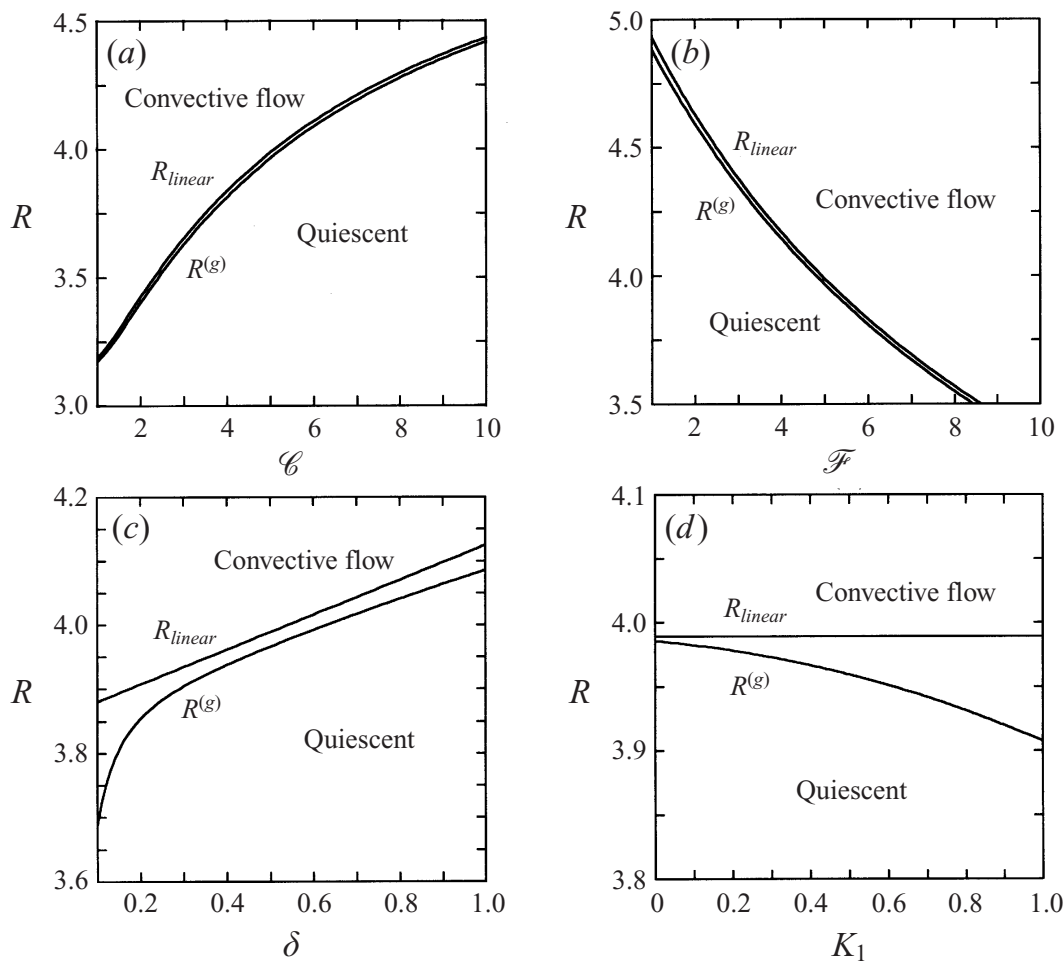

FIGURE 7. The variations of the global critical Rayleigh number $R^{(g)}$ and the linear critical Rayleigh number $R_{\text {linear }}$ versus different physical parameters. In each part $K_{2}=0$ and one parameter varies while the others are fixed. (a) Varying $\mathscr{C}, \mathscr{F}=5, \delta=0.5, K_{1}=0.4 ;(b)$ varying $\mathscr{F}, \mathscr{C}=5, \delta=0.5$, $K_{1}=0.4 ;(c)$ varying $\delta, \mathscr{C}=5, \mathscr{F}=5, K_{1}=0.4 ;(d)$ varying $K_{1}, \mathscr{C}=5, \mathscr{F}=5, \delta=0.5$.

of the onset of the plume convection as

$$
R_{T J J}=\frac{g \gamma \Delta T_{m} H \Pi_{0}}{\kappa \mu}=25,
$$

in which $\gamma=\rho_{0}\left(\alpha^{*}-\beta^{*} / \Gamma\right), \Delta T_{m}$ is the temperature difference between the top and the bottom of the mush, and $H$ is the depth of the mush. We compare (69) with the Rayleigh number $R_{m}$ of $(5 c)$ and note that $\Delta T_{m} / \Gamma \Delta C=O(1)$ and $H /(\kappa / V)=\delta \approx 1$, yielding

$$
\frac{R_{T J J}}{R_{m}}=\frac{\Delta T_{m} H}{\Gamma \Delta C \kappa / V}=O(1) .
$$

From (60) we can compute the global Rayleigh number for the $28 \%$ ammonium chloride solution $\left(\mathscr{C} \approx 9, \mathscr{F} \approx 3.2, \delta \approx 1\right.$ and assume $\left.K_{1}=0.1, K_{2}=0\right)$ and obtain $R^{(g)}=4.6873$. Substituting this into $(11 c)$ we obtain the global critical Rayleigh number for the mush.

$$
R_{m}^{(g)}=20 .
$$

By comparing (69) and (71), we obtain $R_{T J J} / R_{M}^{(g)}=1.25$, which is consistent with the estimation shown in (70). Equation (71) also illustrates the reasonable outcome that the theoretical prediction of the critical Rayleigh number, (71), is lower than the experimental one, (69), because in the experiment the system is already beyond the condition of onset when the plume is observed. 
We also computed the critical Rayleigh number based on the mathematical model considered by A\&W. The result is $R_{m}=30$, which shows again that the imposition of the impermeable boundary condition on the melt/mush interface leads to a more stable state of mush.

\section{Concluding remarks}

We have employed a weakly nonlinear analysis to investigate the stability characteristics of convection in a mush with special attention paid to the stability of the three-dimensional hexagon, which corresponds to the onset of plume convection. We extended the analyses of $\mathrm{A} \& \mathrm{H}$ and $\mathrm{A} \& \mathrm{~W}$, by considering a mathematical model including the more realistic constant-pressure (or permeable) boundary condition at the melt/mush interface. We employed the asymptotic expansion considered by $\mathrm{A} \& \mathrm{~W}$ to analyse the nonlinear behaviour of both the two-dimensional roll and the three-dimensional hexagon convection. Several conclusions are drawn in the following.

(i) The superiority of the constant pressure condition $(8 b)$ has been justified by the present analysis, since the results obtained under this condition show a trend of better agreement with experimental results than those of $A \& H$ and $A \& W$, wherein an impermeable condition is instead employed. This reflects the fact that the vertical flow through the melt/mush interface is of significant influence on the stability in the mush. We therefore infer that by considering the vigorous salt-finger convection above the melt/mush interface (Emms \& Fowler 1994) or by analysing directly the two-layer system (Chen et al. 1994), the analytical results could compare even more favourably with the results of experiment.

(ii) The sense of the flow at the centre of a three-dimensional hexagon depends not only on the factors considered by $\mathrm{A} \& \mathrm{H}$ and $\mathrm{A} \& \mathrm{~W}$, but also on the factors corresponding to the asymmetry of the boundary conditions as well as the finite depth of the mush. The effects from the correction terms corresponding to the finite depth of the mush give a clue that an active mushy layer may allow a down-flow at the centre of the hexagonal cell. This may merit further research in which the near-eutectic approximation, i.e. $\delta \ll 1$, is lifted.

(iii) We find from the analysis with respect to the variation of solid fraction that, if $\mathscr{C}$ is small the minimum solid fraction occurs at the top of the mush, implying that the channel of the plume is initiated from the top of the mush and grows gradually downwards into the mush. If $\mathscr{C}$ is large, the minimum solid fraction may occur within the mush, suggesting that the channel may be initiated from the interior of the mush and grow upwards through only part of the mush. This scenario is supported by the results obtained by Schulze \& Worster (1999) who implemented a nonlinear computation for the two-dimensional roll in the mush.

(iv) From the analysis of the amplitude equation, we find that the stability of the three-dimensional hexagon is invariably subcritical because $R^{(g)}<R_{\text {linear }}$. The variations of $R^{(g)}$ and $R_{\text {linear }}$ with $\mathscr{F}$ and $\mathscr{C}$ have the same trend and the difference between $R^{(g)}$ and $R_{\text {linear }}$ is generally small. Regarding the effects due to $\delta$ and $k_{1}$, the difference between $R^{(g)}$ and $R_{\text {linear }}$ is larger, which provides a good opportunity for the more accurate design of a manufacturing process for the solidification of industrial castings without freckles.

The financial support from National Science Council of Taiwan through Grant NSC 88-2212-E-002-018 is gratefully acknowledged. 


\section{Appendix A. The perturbation equations}

The perturbation equations of different order of magnitude resulting from the weakly nonlinear analysis in $\S 4$ are as follows.

$O\left(\varepsilon^{0} \delta^{0}\right)$ :

$$
\begin{gathered}
\nabla^{2} \theta_{00}-R_{00} w_{00}=0, \quad \frac{\partial}{\partial z} \phi_{00}-\frac{R_{00}}{\bar{C}} w_{00}=0, \\
\nabla^{2} w_{00}+R_{00} \nabla_{H}^{2} \theta_{00}=0, \quad \nabla^{2} u_{00}-R_{00} \frac{\partial^{2}}{\partial x \partial z} \theta_{00}=0, \\
\nabla^{2} v_{00}-R_{00} \frac{\partial^{2}}{\partial y \partial z} \theta_{00}=0 .
\end{gathered}
$$

$O\left(\varepsilon^{0} \delta^{1}\right):$

$$
\begin{gathered}
\nabla^{2} \theta_{01}-R_{00} w_{01}=\left(R_{01}+R_{00} \theta_{B 1}^{\prime}\right) w_{00}-\Omega \frac{\partial}{\partial z} \theta_{00}+\left(\frac{\Omega-1}{\Omega}\right) \frac{\partial}{\partial z}\left(\theta_{B 0} \phi_{00}\right), \\
\frac{\partial}{\partial z} \phi_{01}-\frac{R_{00}}{\bar{C}} w_{01}=\frac{\left(R_{01}+R_{00} \theta_{B 1}^{\prime}\right)}{\bar{C}} w_{00}-\frac{\Omega}{\bar{C}} \frac{\partial}{\partial z} \theta_{00}+\frac{1}{\bar{C}} \frac{\partial}{\partial z}\left(\theta_{B 0} \phi_{00}\right), \\
\nabla^{2} w_{01}+R_{00} \nabla_{H}^{2} \theta_{01}=-R_{01} \nabla_{H}^{2} \theta_{00}+K_{1} \frac{\partial}{\partial z}\left({\overline{\phi^{\prime}}}_{B 0} w_{00}\right)-K_{1} \nabla^{2}\left(\bar{\phi}_{B 0} w_{00}\right), \\
\nabla^{2} u_{01}-R_{00} \frac{\partial^{2}}{\partial x \partial z} \theta_{01}=R_{01} \frac{\partial^{2}}{\partial x \partial z} \theta_{00}+K_{1} \frac{\partial}{\partial x}\left({\overline{\phi^{\prime}}}_{B 0} W_{00}\right)-K_{1} \nabla^{2}\left(\bar{\phi}_{B 0} u_{00}\right), \\
\nabla^{2} v_{01}-R_{00} \frac{\partial^{2}}{\partial y \partial z} \theta_{01}=R_{01} \frac{\partial^{2}}{\partial y \partial z} \theta_{00}+K_{1} \frac{\partial}{\partial y}\left({\overline{\phi^{\prime}}}_{B 0} W_{00}\right)-K_{1} \nabla^{2}\left(\bar{\phi}_{B 0} v_{00}\right) .
\end{gathered}
$$

$O\left(\varepsilon^{1} \delta^{0}\right):$

$$
\begin{gathered}
\nabla^{2} \theta_{10}-R_{00} w_{10}=R_{10} w_{00}+R_{00} \boldsymbol{u}_{00} \cdot \nabla \theta_{00}, \\
\frac{\partial}{\partial z} \phi_{10}-\frac{R_{00}}{\bar{C}} w_{10}=\frac{R_{10}}{\bar{C}} w_{00}+\frac{R_{00}}{\bar{C}} \boldsymbol{u}_{00} \cdot \nabla \theta_{00}, \\
\nabla^{2} w_{10}+R_{00} \nabla_{H}^{2} \theta_{10}=-R_{10} \nabla_{H}^{2} \theta_{00}+\frac{K_{1}}{\Omega} \frac{\partial}{\partial z}\left(\boldsymbol{u}_{00} \cdot \nabla \phi_{00}\right)-\frac{K_{1}}{\Omega} \nabla^{2}\left(\phi_{00} w_{00}\right), \\
\nabla^{2} u_{10}-R_{00} \frac{\partial^{2}}{\partial x \partial z} \theta_{10}=R_{10} \frac{\partial^{2}}{\partial x \partial z} \theta_{00}+\frac{K_{1}}{\Omega} \frac{\partial}{\partial x}\left(\boldsymbol{u}_{00} \cdot \nabla \phi_{00}\right)-\frac{K_{1}}{\Omega} \nabla^{2}\left(\phi_{00} u_{00}\right), \\
\nabla^{2} v_{10}-R_{00} \frac{\partial^{2}}{\partial y \partial z} \theta_{10}=R_{10} \frac{\partial^{2}}{\partial y \partial z} \theta_{00}+\frac{K_{1}}{\Omega} \frac{\partial}{\partial y}\left(\boldsymbol{u}_{00} \cdot \nabla \phi_{00}\right)-\frac{K_{1}}{\Omega} \nabla^{2}\left(\phi_{00} v_{00}\right) .
\end{gathered}
$$


$O\left(\varepsilon^{1} \delta^{1}\right):$

$$
\begin{aligned}
\nabla^{2} \theta_{11}-R_{00} w_{11}= & \left(R_{01}+R_{00} \theta_{B 1}^{\prime}\right) w_{10}+R_{10} w_{01}+\left(R_{11}+R_{10} \theta_{B 1}^{\prime}\right) w_{00} \\
& +R_{00}\left(\boldsymbol{u}_{00} \cdot \nabla \theta_{01}+\boldsymbol{u}_{01} \cdot \nabla \theta_{00}\right)+R_{01} \boldsymbol{u}_{00} \cdot \nabla \theta_{00} \\
& +\left(\frac{\Omega-1}{\Omega}\right) \frac{\partial}{\partial z}\left(\theta_{00} \phi_{00}+\theta_{B 0} \phi_{10}\right)-\Omega \frac{\partial}{\partial z} \theta_{10} \\
\bar{C} \frac{\partial}{\partial z} \phi_{11}-R_{00} w_{11}= & \left(R_{01}+R_{00} \theta_{B 1}^{\prime}\right) w_{10}+R_{10} w_{01}+\left(R_{11}+R_{10} \theta_{B 1}^{\prime}\right) w_{00} \\
& +R_{00}\left(\boldsymbol{u}_{00} \cdot \nabla \theta_{01}+\boldsymbol{u}_{01} \cdot \nabla \theta_{00}\right)+R_{01} \boldsymbol{u}_{00} \cdot \nabla \theta_{00} \\
& +\frac{\partial}{\partial z}\left(\theta_{00} \phi_{00}+\theta_{B 0} \phi_{10}\right)-\Omega \frac{\partial}{\partial z} \theta_{10} \\
\nabla^{2} w_{11}+R_{00} \nabla_{H}^{2} \theta_{11}= & -R_{11} \nabla_{H}^{2} \theta_{00}-R_{10} \nabla_{H}^{2} \theta_{01}-R_{01} \nabla_{H}^{2} \theta_{10} \\
& +\frac{K_{1}}{\Omega} \frac{\partial}{\partial z}\left(\Omega \bar{\phi}_{B 0}^{\prime} w_{10}+\boldsymbol{u}_{01} \cdot \nabla \phi_{00}+\boldsymbol{u}_{00} \cdot \nabla \phi_{01}\right) \\
& +\frac{2 K_{2}}{\Omega} \frac{\partial}{\partial z}\left[\boldsymbol{u}_{00} \cdot \nabla\left(\bar{\phi}_{B 0} \phi_{00}\right)\right] \\
& -\frac{K_{1}}{\Omega} \nabla^{2}\left(\Omega \bar{\phi}_{B 0} w_{10}+\phi_{00} w_{01}+\phi_{01} w_{00}\right) \\
& -\frac{2 K_{2}}{\Omega} \nabla^{2}\left(\bar{\phi}_{B 0} \phi_{00} w_{00}\right) \\
&
\end{aligned}
$$

$$
\begin{aligned}
\nabla^{2} u_{11}-R_{00} \frac{\partial^{2}}{\partial x \partial z} \theta_{11}= & R_{11} \frac{\partial^{2}}{\partial x \partial z} \theta_{00}+R_{10} \frac{\partial^{2}}{\partial x \partial z} \theta_{01}+R_{01} \frac{\partial^{2}}{\partial x \partial z} \theta_{10} \\
& +\frac{K_{1}}{\Omega} \frac{\partial}{\partial x}\left(\Omega \bar{\phi}_{B 0}^{\prime} w_{10}+\boldsymbol{u}_{01} \cdot \nabla \phi_{00}+\boldsymbol{u}_{00} \cdot \nabla \phi_{01}\right) \\
& +\frac{2 K_{2}}{\Omega} \frac{\partial}{\partial x}\left[\boldsymbol{u}_{00} \cdot \nabla\left(\bar{\phi}_{B 0} \phi_{00}\right)\right] \\
& -\frac{K_{1}}{\Omega} \nabla^{2}\left(\Omega \bar{\phi}_{B 0} u_{10}+\phi_{00} u_{01}+\phi_{01} u_{00}\right) \\
& -\frac{2 K_{2}}{\Omega} \nabla^{2}\left(\bar{\phi}_{B 0} \phi_{00} u_{00}\right)
\end{aligned}
$$

$$
\begin{aligned}
\nabla^{2} v_{11}-R_{00} \frac{\partial^{2}}{\partial y \partial z} \theta_{11}= & R_{11} \frac{\partial^{2}}{\partial y \partial z} \theta_{00}+R_{10} \frac{\partial^{2}}{\partial y \partial z} \theta_{01}+R_{01} \frac{\partial^{2}}{\partial y \partial z} \theta_{10} \\
& +\frac{K_{1}}{\Omega} \frac{\partial}{\partial y}\left(\Omega \bar{\phi}_{B 0}^{\prime} w_{10}+\boldsymbol{u}_{01} \cdot \nabla \phi_{00}+\boldsymbol{u}_{00} \cdot \nabla \phi_{01}\right) \\
& +\frac{2 K_{2}}{\Omega} \frac{\partial}{\partial y}\left[\boldsymbol{u}_{00} \cdot \nabla\left(\bar{\phi}_{B 0} \phi_{00}\right)\right] \\
& -\frac{K_{1}}{\Omega} \nabla^{2}\left(\Omega \bar{\phi}_{B 0} v_{10}+\phi_{00} v_{01}+\phi_{01} v_{00}\right) \\
& -\frac{2 K_{2}}{\Omega} \nabla^{2}\left(\bar{\phi}_{B 0} \phi_{00} v_{00}\right) .
\end{aligned}
$$


$O\left(\varepsilon^{2} \delta^{0}\right):$

$$
\begin{aligned}
& \nabla^{2} \theta_{20}-R_{00} w_{20}=R_{20} w_{00}+R_{10} w_{10}+R_{10} \boldsymbol{u}_{00} \cdot \nabla \theta_{00}+R_{00}\left(\boldsymbol{u}_{00} \cdot \nabla \theta_{10}+\boldsymbol{u}_{10} \cdot \nabla \theta_{00}\right) \\
& +\left(1-\frac{1}{\Omega}\right)\left[\frac{\partial}{\partial z}\left(\theta_{B 0} \phi_{2(-1)}\right)-\theta_{B 0} \frac{\partial \phi_{00}}{\partial \tau}\right]+\Omega \frac{\partial \theta_{00}}{\partial \tau}, \\
& \bar{C} \frac{\partial}{\partial z} \phi_{20}-R_{00} w_{20}=R_{20} w_{00}+R_{10} w_{10}+R_{10} \boldsymbol{u}_{00} \cdot \nabla \theta_{00} \\
& +R_{00}\left(\boldsymbol{u}_{00} \cdot \nabla \theta_{10}+\boldsymbol{u}_{10} \cdot \nabla \theta_{00}\right)+\frac{\partial}{\partial z}\left(\theta_{B 0} \phi_{2(-1)}\right) \\
& -\theta_{B 0} \frac{\partial \phi_{00}}{\partial \tau}+\bar{C} \frac{\partial \phi_{01}}{\partial \tau}+\Omega \frac{\partial \theta_{00}}{\partial \tau} \\
& \nabla^{2} w_{20}+R_{00} \nabla_{H}^{2} \theta_{20}=\frac{K_{1}}{\Omega}\left[\frac{\partial}{\partial z}\left(\boldsymbol{u}_{10} \cdot \nabla \phi_{00}+\boldsymbol{u}_{00} \cdot \nabla \phi_{10}\right)-\nabla^{2}\left(\phi_{00} w_{10}+\phi_{10} w_{00}\right)\right] \\
& +\frac{K_{2}}{\Omega^{2}}\left[\frac{\partial}{\partial z}\left(\boldsymbol{u}_{00} \cdot \nabla \phi_{00}^{2}\right)-\nabla^{2}\left(\phi_{00}^{2} w_{00}\right)\right] \\
& -R_{20} \nabla_{H}^{2} \theta_{00}-R_{10} \nabla_{H}^{2} \theta_{10} \\
& \nabla^{2} u_{20}-R_{00} \frac{\partial}{\partial x \partial z} \theta_{20}=\frac{K_{1}}{\Omega}\left[\frac{\partial}{\partial x}\left(\boldsymbol{u}_{10} \cdot \nabla \phi_{00}+\boldsymbol{u}_{00} \cdot \nabla \phi_{10}\right)-\nabla^{2}\left(\phi_{00} u_{10}+\phi_{10} u_{00}\right)\right] \\
& +\frac{K_{2}}{\Omega^{2}}\left[\frac{\partial}{\partial x}\left(\boldsymbol{u}_{00} \cdot \nabla \phi_{00}^{2}\right)-\nabla^{2}\left(\phi_{00}^{2} u_{00}\right)\right] \\
& +R_{20} \frac{\partial^{2}}{\partial x \partial z} \theta_{00}+R_{10} \frac{\partial^{2}}{\partial x \partial z} \theta_{10} \\
& \nabla^{2} v_{20}-R_{00} \frac{\partial^{2}}{\partial y \partial z} \theta_{20}=\frac{K_{1}}{\Omega}\left[\frac{\partial}{\partial y}\left(\boldsymbol{u}_{10} \cdot \nabla \phi_{00}+\boldsymbol{u}_{00} \cdot \nabla \phi_{10}\right)-\nabla^{2}\left(\phi_{00} v_{10}+\phi_{10} v_{00}\right)\right] \\
& +\frac{K_{2}}{\Omega^{2}}\left[\frac{\partial}{\partial y}\left(\boldsymbol{u}_{00} \cdot \nabla \phi_{00}^{2}\right)-\nabla^{2}\left(\phi_{00}^{2} v_{00}\right)\right] \\
& +R_{20} \frac{\partial^{2}}{\partial y \partial z} \theta_{00}+R_{10} \frac{\partial^{2}}{\partial y \partial z} \theta_{10} .
\end{aligned}
$$

$O\left(\varepsilon^{2} \delta^{1}\right):$

$$
\begin{aligned}
\nabla^{2} \theta_{21}-R_{00} w_{21}= & R_{21} w_{00}+R_{20}\left(w_{01}+\theta_{B 1}^{\prime} w_{00}\right)+R_{11} w_{10}+R_{10}\left(w_{11}+\theta_{B 1}^{\prime} w_{10}\right) \\
& +\left(R_{01}+R_{00} \theta_{B 1}^{\prime}\right) w_{20}-\Omega \frac{\partial \theta_{20}}{\partial z}+R_{00} \boldsymbol{u}_{00} \cdot \nabla \theta_{11} \\
& +\left(R_{00} \boldsymbol{u}_{01}+R_{01} \boldsymbol{u}_{00}\right) \cdot \nabla \theta_{10}+\left(R_{00} \boldsymbol{u}_{10}+R_{10} \boldsymbol{u}_{00}\right) \cdot \nabla \theta_{01} \\
& +\left(R_{00} \boldsymbol{u}_{11}+R_{01} \boldsymbol{u}_{10}+R_{10} \boldsymbol{u}_{01}+R_{11} \boldsymbol{u}_{00}\right) \cdot \nabla \theta_{00} \\
& +\left(1-\frac{1}{\Omega}\right) \frac{\partial}{\partial z}\left(\theta_{B 1} \phi_{2(-1)}+\theta_{B 0} \phi_{20}+\theta_{00} \phi_{10}+\theta_{10} \phi_{00}\right) \\
& -\left(1-\frac{1}{\Omega}\right)\left(\theta_{B 0} \frac{\partial \phi_{01}}{\partial \tau}+\theta_{B 1} \frac{\partial \phi_{00}}{\partial \tau}\right)+\Omega \frac{\partial \theta_{01}}{\partial \tau}-\frac{\bar{F}_{\bar{C}}}{\bar{C}} \bar{\phi}_{B 0} \frac{\partial \theta_{00}}{\partial \tau},
\end{aligned}
$$


80 C. A. Chung and F. Chen

$$
\begin{aligned}
& \bar{C} \frac{\partial}{\partial z} \phi_{21}-R_{00} w_{21}=R_{21} w_{00}+R_{20}\left(w_{01}+\theta_{B 1}^{\prime} w_{00}\right)+R_{11} w_{10}+R_{10}\left(w_{11}+\theta_{B 1}^{\prime} w_{10}\right) \\
& +\left(R_{01}+R_{00} \theta_{B 1}^{\prime}\right) w_{20}-\Omega \frac{\partial \theta_{20}}{\partial z}+R_{00} \boldsymbol{u}_{00} \cdot \nabla \theta_{11} \\
& +\left(R_{00} \boldsymbol{u}_{01}+R_{01} \boldsymbol{u}_{00}\right) \cdot \nabla \theta_{10}+\left(R_{00} \boldsymbol{u}_{10}+R_{10} \boldsymbol{u}_{00}\right) \cdot \nabla \theta_{01} \\
& +\left(R_{00} \boldsymbol{u}_{11}+R_{01} \boldsymbol{u}_{10}+R_{10} \boldsymbol{u}_{01}+R_{11} \boldsymbol{u}_{00}\right) \cdot \nabla \theta_{00} \\
& +\frac{\partial}{\partial z}\left(\theta_{B 1} \phi_{2(-1)}+\theta_{B 0} \phi_{20}+\theta_{00} \phi_{10}+\theta_{10} \phi_{00}\right) \\
& -\theta_{B 0} \frac{\partial \phi_{01}}{\partial \tau}-\theta_{B 1} \frac{\partial \phi_{00}}{\partial \tau}+\Omega \frac{\partial \theta_{01}}{\partial \tau}-\Omega \bar{\phi}_{B 0} \frac{\partial \theta_{00}}{\partial \tau}+\bar{C} \frac{\partial \phi_{02}}{\partial \tau} \\
& \nabla^{2} w_{21}+R_{00} \nabla_{H}^{2} \theta_{21}=-R_{21} \nabla_{H}^{2} \theta_{00}-R_{01} \nabla_{H}^{2} \theta_{20}-R_{11} \nabla_{H}^{2} \theta_{10} \\
& -R_{10} \nabla_{H}^{2} \theta_{11}-R_{01} \nabla_{H}^{2} \theta_{20} \\
& +\frac{K_{1}}{\Omega} \frac{\partial}{\partial z}\left(\Omega \bar{\phi}_{B 0}^{\prime} w_{20}+\boldsymbol{u}_{11} \cdot \nabla \phi_{00}+\boldsymbol{u}_{10} \cdot \nabla \phi_{01}\right. \\
& \left.+\boldsymbol{u}_{01} \cdot \nabla \phi_{10}+\boldsymbol{u}_{00} \cdot \nabla \phi_{11}\right) \\
& +\frac{K_{2}}{\Omega^{2}} \frac{\partial}{\partial z}\left[2 \Omega\left(\boldsymbol{u}_{10} \cdot \nabla\left(\bar{\phi}_{B 0} \phi_{00}\right)+\boldsymbol{u}_{00} \cdot \nabla\left(\bar{\phi}_{B 0} \phi_{10}\right)\right)\right. \\
& \left.+2 \boldsymbol{u}_{00} \cdot \nabla\left(\phi_{00} \phi_{01}\right)+\boldsymbol{u}_{01} \cdot \nabla \phi_{00}^{2}\right] \\
& +\frac{3 K_{3}}{\Omega^{2}} \frac{\partial}{\partial z}\left[\boldsymbol{u}_{00} \cdot \nabla\left(\bar{\phi}_{B 0} \phi_{00}^{2}\right)\right]-\frac{K_{1}}{\Omega} \nabla^{2}\left(\Omega \bar{\phi}_{B 0} w_{20}+\phi_{00} w_{11}\right. \\
& \left.+\phi_{01} w_{10}+\phi_{10} w_{01}+\phi_{11} w_{00}\right) \\
& -\frac{K_{2}}{\Omega^{2}} \nabla^{2}\left[2 \Omega \bar{\phi}_{B 0}\left(\phi_{00} w_{10}+\phi_{10} w_{00}\right)+2 \phi_{00} \phi_{01} w_{00}+\phi_{00}^{2} w_{01}\right] \\
& -\frac{3 K_{3}}{\Omega^{2}} \nabla^{2}\left(\bar{\phi}_{B 0} \phi_{00}^{2} w_{00}\right)
\end{aligned}
$$

\section{Appendix B. The coefficients for $W_{01}$}

The coefficients of (63) for $W_{01}$ are

$$
\begin{aligned}
& \alpha^{(0)}=2.5120 \frac{\bar{F}}{\bar{C}^{2} \Omega}, \quad \alpha_{c}^{(0)}=-1.6282 \frac{\bar{F}}{\bar{C}^{2} \Omega}, \quad \alpha_{c}^{(1)}=-0.2183 \frac{\bar{F}}{\bar{C}^{2} \Omega}+2.9708 \Omega, \\
& \alpha_{c}^{(2)}=2.5879 \frac{\bar{F}}{\bar{C}^{2} \Omega}-2.5879 \Omega, \quad \alpha_{s}^{(0)}=-53.9436 \frac{\bar{F}}{\bar{C}^{2} \Omega}-1.7219 \Omega, \\
& \alpha_{s}^{(1)}=-0.1063 \frac{\bar{F}}{\bar{C}^{2} \Omega}+0.9999 \Omega, \quad \alpha_{c h}^{(0)}=-0.8839 \frac{\bar{F}}{\bar{C}^{2} \Omega}, \\
& \alpha_{c h}^{(1)}=-0.002159 \frac{\bar{F}}{\bar{C}^{2} \Omega}+0.02938 \Omega, \quad \alpha_{c h}^{(2)}=0.02560 \frac{\bar{F}}{\bar{C}^{2} \Omega}-0.02560 \Omega,
\end{aligned}
$$




$$
\alpha_{s h}^{(0)}=0.03640 \frac{\bar{F}}{\bar{C}^{2} \Omega}-0.03136 \Omega, \quad \alpha_{s h}^{(1)}=-0.01158 \frac{\bar{F}}{\bar{C}^{2} \Omega}+0.006115 \Omega \text {. }
$$

\section{Appendix C. The coefficients for $\Phi_{01}$}

The coefficients of (68) for $\phi_{01}$ are

$$
\begin{aligned}
& \beta^{(0)}=\frac{1}{\bar{C}}\left(\frac{10.4652}{\bar{C}}+112.1426 \frac{\bar{F}}{\bar{C}^{2} \Omega}+4.9528 \Omega\right), \\
& \beta^{(1)}=\frac{1}{\bar{C}}\left(-\frac{10.4652}{\bar{C}}+13.0765 \frac{\bar{F}}{\bar{C}^{2} \Omega}\right) \text {, } \\
& \beta_{c}^{(0)}=\frac{1}{\bar{C}}\left(\frac{8.9000}{\bar{C}}+104.0695 \frac{\bar{F}}{\bar{C}^{2} \Omega}+0.9930 \Omega\right), \\
& \beta_{c}^{(1)}=\frac{1}{\bar{C}}\left(\frac{-8.9000}{\bar{C}}+4.2363 \frac{\bar{F}}{\bar{C}^{2} \Omega}+2.8661 \Omega\right) \text {, } \\
& \beta_{s}^{(0)}=\frac{1}{\bar{C}}\left(-4.9118 \frac{\bar{F}}{\bar{C}^{2} \Omega}+0.7947 \Omega\right), \quad \beta_{s}^{(1)}=\frac{1}{\bar{C}}\left(-0.4390 \frac{\bar{F}}{\bar{C}^{2} \Omega}+5.9754 \Omega\right), \\
& \beta_{s}^{(2)}=\frac{5.2053}{\bar{C}}\left(\frac{\bar{F}}{\bar{C}^{2} \Omega}-\Omega\right), \quad \beta_{c h}^{(0)}=\frac{1}{\bar{C}}\left(-\frac{0.08803}{\bar{C}}+0.08806 \frac{\bar{F}}{\bar{C}^{2} \Omega}-0.0004638 \Omega\right), \\
& \beta_{c h}^{(1)}=\frac{1}{\bar{C}}\left(-\frac{0.08803}{\bar{C}}-0.02961 \frac{\bar{F}}{\bar{C}^{2} \Omega}-0.06521 \Omega\right), \\
& \beta_{s h}^{(0)}=\frac{1}{\bar{C}}\left(-1.0921 \frac{\bar{F}}{\bar{C}^{2} \Omega}-0.01485 \Omega\right), \quad \beta_{s h}^{(1)}=\frac{1}{\bar{C}}\left(-0.002685 \frac{\bar{F}}{\bar{C}^{2} \Omega}+0.03654 \Omega\right), \\
& \beta_{s h}^{(2)}=\frac{0.03183}{\bar{C}}\left(\frac{\bar{F}}{\bar{C}^{2} \Omega}-\Omega\right) \text {. }
\end{aligned}
$$

\section{REFERENCES}

Amberg, G. \& Homsy, G. M. 1993 Nonlinear analysis of buoyant convection in binary solidification with application to channel formation. J. Fluid Mech. 252, 79-98 (referred to herein as A\&H).

ANDERson, D. M. \& Worster, M. G. 1995 Weakly nonlinear analysis of convection in a mushy layer during the solidification of binary alloys. J. Fluid. Mech. 302, 307-331 (referred to herein as $\mathrm{A} \& \mathrm{~W})$.

Brattkus, K. \& Davis, S. H. 1988 Cellular growth near absolute stability. Phys. Rev. B 38, $11452-11460$

Chen, C. F. 1995 Experimental study of convection in a mushy layer during directional solidification J. Fluid Mech. 293, 81-98.

Chen, F. 1997 Formation of double-diffusive layers in the directional solidification of binary solution. J. Cryst. Growth 179, 277-286.

CHen, F. \& CHEN, C. F. 1991 Experimental study of directional solidification of aqueous ammonium chloride solution. J. Fluid Mech. 227, 567-586.

Chen, F., Lu, J. W. \& YANG, T. L. 1994 Convection instability in ammonium chloride solution directionally solidified from below. J. Fluid Mech. 276, 163-187. 
Chinarel A. O. P. \& Worster M. G. 1995 Flow focusing instability in a solidifing mushy layer. J. Fluid Mech. 297, 293-305.

Copley, S. M., Giamei, A. F., Johnson, S. M. \& Hornbecker, M. F. 1970 The origin of freckles in unidirectionally solidified castings. Metall. Trans. 1, 2193-2204.

Emms, P. W. \& Fowler, A. C. 1994 Compositional convection in the solidification of binary alloys J. Fluid Mech. 262, 111-139.

Fowler, A. G. 1985 The formation of freckles in binary alloys. IMA J. Appl. Maths 35, 159-174.

Kuske, R. \& MatKowsky, B. J. 1994 On roll, square and hexagonal cellular flames. Eur. J. Appl. Maths 5, 65-93.

Sample, A. K. \& Hellawel, A. 1984 The mechanism of formation and prevention of channel segregation during alloy solidification. Metall. Trans. A 15, 2163-2173.

Schulze, T. P. \& Worster, M. G. 1999 Weak convection, liquid inclusions and the formation of chimneys in mushy layers. J. Fluid Mech. 388, 197-215.

SEgEL, L. A. 1965 Distant side-walls cause slow amplitude modulation of cellular convection. J. Fluid Mech. 38, 203-224.

TAit, S., Jahrling, K. \& Jaupart, C. 1992 The planform of compositional convection and chimney formation in a mushy layer. Nature 359, 406-408.

TAIT, S. \& JAUPART, C. 1992 Compositional convection in a reactive crystalline mush and melt differentiation. J. Geophys. Res. 97 (B 5), 6735-6759.

Worster, M. G. 1992 Instabilities of the liquid and mushy regions during solidification of alloys. J. Fluid Mech. 237, 649-669.

Worster, M. G. 1997 Convection in mushy layers. Ann. Rev. Fluid Mech. 29, 91-122.

Worster, M. G. \& Kerr, R. C. 1994 The transient behavior of alloys solidified from below prior to the formation of chimneys. J. Fluid Mech. 269, 23-44. 